\title{
Stability of Quantum Eigenstates and Collapse of Superposition of States in a Fluctuating Vacuum: The Madelung Hydrodynamic Approach
}

\author{
P. Chiarelli and S. Chiarelli
}

\section{ABSTRACT}

The paper investigates the quantum fluctuating dynamics by using the stochastic generalization of the Madelung quantum-hydrodynamic approach. By using the discrete approach, the path integral solution is derived in order to investigate how the final stationary configuration is obtained from the initial quantum superposition of states. The model shows that the quantum eigenstates remain stationary configurations with a very small perturbation of their mass density distribution and that any eigenstate, contributing to a quantum superposition of states, can be reached in the final stationary configuration. When the non-local quantum potential acquires a finite range of interaction, the work shows that the macroscopic coarse-grained description of the theory can lead to a really classical system. The minimum uncertainty attainable in the stochastic Madelung model is shown to be compatible with maximum speed of transmission of information and interactions. The theory shows that, in the quantum deterministic limit, the uncertainty relations of quantum mechanics are obtained. The connections with the decoherence theory and the Copenhagen interpretation of quantum mechanics are also discussed.

Published Online: September 10, 2021

ISSN: $2684-4451$

DOI :10.24018/ejphysics.2021.3.5.97

P. Chiarelli *

National Council of Research of Italy, Pisa, Italy.

Interdepartmental Center "E. Piaggio",

Faculty of Engineering, University of Pisa, Pisa, Italy.

(e-mail: pchiare@ifc.cnr.it)

S. Chiarelli

Scuola Normale Superiore, Pisa, Italy;

(e-mail: simone.chiarelli@ ${ }^{@}$ sns.it)

*Corresponding Author

Keywords: fluctuation-induced quantum decoherence; mesoscale dynamics Qbits; quantum dissipation; quantum to classical transition; stability of quantum eigenstates; stochastic quantum hydrodynamics; wave function collapse.

\section{INTRODUCTION}

The dependence of the dynamics of bodies on the magnitude of their mass has a disconnection in the passage from the quantum to the classical description [1]-[18]. This lack of unitary description has led to quantum results that contradict our sense of reality [1]-[4]. A quantitative investigation of the problem was provided by Bell [3] in response to the so-called EPR paradox [2] where quantum non-locality is analyzed with respect to the notions of classical freedom and local relativistic causality.

In order to solve this problem, various interpretations of quantum mechanics have been proposed such as the manyworlds interpretation [19], the Bohmian mechanics [20], [21], the modal interpretation [22], the relational interpretation [23], the consistent histories [24], the transactional interpretation [25], [26], the QBism [27], the Madelung quantum hydrodynamics [28]-[30], the decoherence approach [31].

Madelung's model (which is a special case of Bohmian [32] mechanics) that has the important property of being mathematically equivalent to that of Schrödinger [29], treats the evolution of the wave function $\psi=|\psi| e^{i \frac{S}{\hbar}}$ as the motion of the mass density $|\psi|^{2}$ with the momentum $p_{i}=\frac{\partial S}{\partial q_{i}}$ in a classical-like way [29], where the quantum non-locality is generated by the quantum potential, introducing the concept of trajectories and the existence of physical reality independent by the measure.

Madelung's description meets classic mechanics as soon as the Planck constant is set to zero. This is in accordance with the widely accepted concept that quantum mechanics converges to classical mechanics for objects whose mass is of macroscopic units for which the Planck's constant is infinitesimal.

Thus, Madelung's approach represents a good theoretical framework for describing the quantum to classical transition, mesoscale phenomena and the interaction between quantum and classical systems [33]-[35].

For the sake of truth, it should be noted that even if Planck's constant is small (as in the macroscopic dynamics), if we cancel it with the associated quantum potential from the quantum hydrodynamics equations, we also eliminate the quantum stationary eigenstates, and we profoundly change the nature of the equations of motion (see appendix B in ref. [36]). Hence, a well-defined mathematical procedure is needed to correctly pass from the non-local quantum 
description to the classical one [37] in the very small Planck constant limit.

Other aspects of open quantum evolution are captured by the decoherence approach which investigates the possibility of obtaining the macroscopic state through the loss of the quantum wave coherence generated by the presence of the environment [38]-[41]. Also, from the experimental and numerical simulation point of view, there is evidence that the decoherence and localization of quantum states can be generated by stochastic fluctuations [38], [41].

Recently [42], the author demonstrated that, if we consider the non-static foundations of the universe, as suggested by De Sitter, with minimal space-time oscillations and fluctuations of the mass density of the vacuum, Madelung's description leads to the stochastic quantum hydrodynamic model (SQHM) which in the limit of microscopic systems (i.e., whose physical size is smaller than the De Broglie length) leads to the Langevin-Schrodinger equation.

In the limit of zero fluctuations, where De Broglie's length becomes infinite, the SQHM reduces to quantum mechanics which represents the deterministic limit of stochastic theory.

The stochastic quantum hydrodynamics shows that, in the large-scale coarse-grained description, depending on the type of Hamiltonian potential, there is the possibility that the range of interaction of the quantum potential becomes finite leading to the emergence of the classical description on infinitely larger scale [42].

\section{A. Fundamental Issue}

The main issue of the work is to investigate the irreversible quantum dynamics, originating from the Madelung-Bohm quantum potential, when stochastic fluctuations of the mass density are present in the space-time. The novelty, here, is that the noise arises from random fluctuations of the background metric (i.e., gravitational fluctuations) but not from an external environment. Thus, the quantum irreversible behavior can be seen as an intrinsic property of the system instead of deriving from something else out of it.

For need of synthesis, the fluctuations of the background metric are not explicitly derived from the gravitational theory, but are given here as a basic statement.

The derivation of quantum mechanics as a stochastic process dates back to Nelson's work [43] and has never been interrupted since. Although Nelson's hypotheses proved unsatisfactory, the definitive answer to the question was given by the integral path representation of quantum mechancis [44] (see Appendix A) which showed that quantum mechanics can be seen as a stochastic process of imaginary time. Such quantum fluctuations are different from the thermal ones since they produce a "reversible" pseudodiffusion process which the Madelung's quantum hydrodynamics shows to be produced by the so-called quantum potential.

In the present work, the authors want to generalize the law of motion when both fluctuations on the imaginary time of quantum mechanics and those on real-time of vacuum mass density are present in space-time.

In a previous related article [42] the authors derived the generalized expression of the Bohm-Madelung quantum potential for the considered problem. In this work the authors further analyze the stochastic quantum hydrodynamic model for describing the quantum irreversible behavior. The issues of this work are:

i. To obtain the integral path solution of the quantumstochastic dynamics for describing the relaxation of the superposition of states;

ii. To investigate the characteristics of the limiting stationary configurations and their connection with the deterministic quantum eigenstates;

iii. To show that the deterministic limit of the theory asymptotically converges to quantum mechanics in microscopic systems;

iv. To derive the conditions under which classical behavior can be realized on large-scale systems;

v. To generalize the uncertainty relations for the quantumstochastic model;

vi. To analyze the connections with the decoherence theory and the Copenhagen interpretation of quantum mechanics.

\section{The STOCHASTIC QUANTUM HYDRODYNAMIC EQUATION}

As shown in reference [42], in presence of vacuum mass density fluctuations, the quantum-hydrodynamic equations of motion acquire the stochastic form

$\dot{q}_{i}=\frac{p}{m}$

$\dot{p}_{j}=-m \kappa \dot{q}_{j_{(t)}}-\frac{1}{m} \frac{\partial\left(V_{(q)}+V_{\left.q u_{(n)}\right)}\right)}{\partial q_{j}}+m \varpi_{j(q, t, T)}$

where

$$
V_{q u_{(n)}}=-\frac{\hbar^{2}}{2 m} \frac{1}{n^{1 / 2}} \frac{\partial^{2} n^{1 / 2}}{\partial q_{\beta} \partial q_{\beta}}
$$

Where $n$ is the probability mass density (PMD). An ansatz for the noise $\varpi_{(q, t, T)}$ is [42]:

$$
\begin{aligned}
& <\varpi_{j\left(q_{\alpha}, t\right)}, \varpi_{i\left(q_{\beta}+\lambda, t+\tau\right)}> \\
& =<\varpi_{j\left(q_{\alpha}\right)}, \varpi_{i\left(q_{\beta}\right)}>_{(T)} F(\lambda) \delta(\tau) \delta_{\alpha \beta} \delta_{j i}
\end{aligned}
$$

i. Gaussian with zero correlation time,

ii. isotropic in space;

iii. not correlated between different coordinates

iv. the shape of the correlation function $F(\lambda)$ reads [42]:

$\lim _{T \rightarrow 0} F(\lambda) \propto \frac{1}{\lambda_{c}} \exp \left[-\left(\frac{\lambda}{\lambda_{c}}\right)^{2}\right]$.

where

$\lambda_{c}=\sqrt{2} \frac{\hbar}{\sqrt{m k T}}$ 
is the De Broglie length and where $\mathrm{T}$ is the fluctuationamplitude parameter (with the dimension of a temperature).

The spatial correlation function $F(\lambda)$ of the noise in vacuum is determined [36], [42] by imposing that mass density (MD) fluctuations in the vacuum are subjected to quantum behavior: Since the quantum potential energy is dependent on the derivatives of the MD, the energy of these fluctuations is greater the shorter their wavelength $\lambda$.

Generally speaking [42], the reduced spatial probability of the phase space mass density of the Smolukowski equation for the Marcovian process (2) [42] (hereinafter we refer to it as probability mass density $n$, is not the mass density of the quantum hydrodynamic description (i.e., $\tilde{n}=|\psi|^{2}$ ): The former one is a probability distribution, the latter one is a stochastic function. Nevertheless, they are connected by the limit convergence $\lim _{T \rightarrow 0} n=\lim _{T \rightarrow 0} \tilde{n}=|\psi|$. Furthermore, as shown in ref. [42], near the deterministic limit the difference, in the quantum potential force, of the two variables, in leading order approximation of velocity field, reads:

$\frac{\partial}{\partial q_{\alpha}}\left(V_{q u_{(n)}}-V_{q u_{(}(\tilde{n})}\right) \cong m \bar{D}^{1 / 2} \xi_{(t)}+A_{1(q, t)} \dot{q}+O\left(\dot{q}^{2}\right)$,

giving rise to Gaussian stochastic force noise with zero mean and drift term proportional to the velocity field $\dot{q}$. If $A_{1}$ it is assumed to be constant, it is equal to the friction coefficient $\beta=m \kappa$ [42] in (2).

Equations (1)-(3) for the description of a system with physical length $\mathcal{L}$, such as $\frac{\lambda_{c}}{\mathcal{L}} \rightarrow \infty$ (i.e., $m \rightarrow 0$ or $T \rightarrow 0$ ), lead to $F(\lambda) \rightarrow 0$ and hence to the canonical quantum mechanics that represents the deterministic limit of the stochastic theory [29].

On the other hand, for macroscopic systems with $\frac{\lambda_{c}}{\mathcal{L}} \rightarrow 0$, the spatial correlation function of noise converges to the $\delta$ function, and its spectrum is white (as expected in the classical vacuum).

For a mesoscale system (i.e., $\frac{\mathcal{L}}{\lambda_{c}} \approx 1$ ), where the quantum non-local interaction is important, the noise at the leading order of approximation in $\frac{\mathcal{L}}{\lambda_{c}}$,-reads [42]:

$\varpi_{(q, t, T)}=\kappa D^{1 / 2} \xi_{(t)}$

where

$\kappa \cong \alpha \frac{2 k T}{m D}+O\left(D^{-2}\right)$

where $\alpha$ is the numerical parameter that measures how the quantum hydrodynamic trajectories of the motion are perturbed by fluctuations (i.e., how easy is to produce quantum decoherence and dissipation into the system). This parameter is specific for the considered system since its sensitivity to fluctuations is correlated to the characteristics of the trajectories of motion. This aspect goes beyond the scope of this work and (9) is given here as a semi-empirical parameter.

$\alpha$ varies from $\alpha=0$ (as given in the deterministic limit of quantum mechanics) to $\alpha=1$ for the so-called "dust matter" to which the MDD converges in the classical limit of the Madelung's quantum hydrodynamics [29].

Thence, for microscopic systems of physical length $\mathcal{L}$, such as $\mathcal{L}<\lambda_{c}$, equations (1-2) read:

$$
\ddot{q}_{j_{(t)}}=-\kappa \dot{q}_{j_{(t)}}-\frac{1}{m} \frac{\partial\left(V_{(q)}+V_{q u}\right)}{\partial q_{j}}+\kappa D_{j k}^{1 / 2} \xi_{k(t)}
$$

that for problems where fast variables (with characteristic time $\tau_{c h}<\approx \frac{1}{\kappa}$ ) are of no physical interest, reduces to [45]:

$\dot{q}_{j_{(t)}}=-\frac{1}{m \kappa} \frac{\partial\left(V_{(q)}+V_{q u}\right)}{\partial q_{j}}+D_{j k}^{1 / 2} \xi_{k(t)}$.

(The existence of stationary long-time asymptotical states given by (11) is warranted by the dissipative force $-\kappa \dot{q}_{(t)}$ that, for Hamiltonian potential $V_{(q)}$ independent by time, leads to the asymptotical condition $\lim _{t \rightarrow \infty}<\ddot{q}_{(t)}>=0$ [45]).

In the case of unidimensional system (11) simplifies to:

$\dot{q}_{(t)}=-\frac{1}{m \kappa} \frac{\partial\left(V_{(q)}+V_{q u}\right)}{\partial q}+D^{1 / 2} \xi_{(t)}$

Moreover, by posing $D$ and $\kappa[42]$ :

$D=\gamma_{D}\left(\frac{\mathcal{L}}{\lambda_{c}}\right)^{p} \frac{\hbar}{2 m}$

$\kappa=2 \alpha \frac{k T}{m D}=4 \alpha \frac{k T}{\gamma_{D} \hbar}\left(\frac{\lambda_{c}}{\mathcal{L}}\right)^{p}$

where $\gamma_{D}$ is a pure number, (10) reads:

$$
\begin{aligned}
\ddot{q}_{(t)}= & -4 \alpha \frac{k T}{\gamma_{D} \hbar}\left(\frac{\lambda_{c}}{\mathcal{L}}\right)^{p} \dot{q}_{(t)}-\frac{1}{m} \frac{\partial\left(V_{(q)}+V_{q u}\right)}{\partial q} \\
& +4 \alpha \frac{k T}{\hbar \sqrt{\gamma_{D}}}\left(\frac{\lambda_{c}}{\mathcal{L}}\right)^{p / 2}\left(\frac{\hbar}{2 m}\right)^{1 / 2} \xi_{(t)}
\end{aligned}
$$

where, for $p \leq 2$, in the deterministic limit of quantum mechanics the "dissipation parameter" $\alpha$, undergoes the condition 
$\lim _{T \rightarrow 0} \alpha=0$

For the description of sufficiently slow kinetics, equation (15) simplifies to:

$\dot{q}_{(t)}=-\frac{\gamma_{D} \hbar}{4 \alpha m k T}\left(\frac{\mathcal{L}}{\lambda_{c}}\right)^{p} \frac{\partial\left(V_{(q)}+V_{q u}\right)}{\partial q}+\sqrt{\gamma_{D}}\left(\frac{\mathcal{L}}{\lambda_{c}}\right)^{p / 2}\left(\frac{\hbar}{2 m}\right)^{1 / 2} \xi_{(t)}$

A physical example, with the diffusion coefficient proportional to $k T$ (obtainable for $p=2$ ), leads to the identities:

$$
\begin{gathered}
D=\frac{2 \alpha k T}{m \kappa}=\gamma_{D}\left(\frac{\mathcal{L}}{\lambda_{c}}\right)^{2} \frac{\hbar}{2 m}=\gamma_{D} \mathcal{L}^{2} \frac{k T}{4 \hbar} \\
\kappa=4 \alpha_{(T)} \frac{k T}{\gamma_{D} \hbar}\left(\frac{\lambda_{c}}{\mathcal{L}}\right)^{2}=\frac{8 \hbar \alpha}{\gamma_{D} m \mathcal{L}^{2}} \\
\ddot{q}_{(t)}=-\frac{8 \hbar \alpha}{\gamma_{D} m \mathcal{L}^{2}} \dot{q}_{(t)}-\frac{1}{m} \frac{\partial\left(V_{(q)}+V_{q u}\right)}{\partial q} \\
+\frac{4 \hbar \alpha}{\sqrt{\gamma_{D}} m \mathcal{L}} \sqrt{\frac{k T}{\hbar}} \xi_{(t)}
\end{gathered}
$$

and, for slow kinetics such as $\tau_{c h} \gg \frac{1}{\kappa}$, to:

$\dot{q}_{(t)}=-\gamma_{D} \frac{\mathcal{L}^{2}}{8 \hbar \alpha} \frac{\partial\left(V_{(q)}+V_{q u}\right)}{\partial q}+\sqrt{\gamma_{D}} \frac{\mathcal{L}}{2} \sqrt{\frac{k T}{\hbar}} \xi_{(t)}$.

For $m \approx 10^{-30} \mathrm{Kg}$ and $\mathcal{L} \approx 10^{-10} \mathrm{~m}$, equation (21), holds for kinetics with characteristic time:

$\tau_{c h} \gg \frac{1}{\kappa}=\frac{\gamma_{D} m \mathcal{L}^{2}}{8 \hbar \alpha} \approx \gamma_{D} \frac{10^{-17} s}{\alpha}$.

The evaluation of the order of magnitude of $\frac{1}{\kappa}$ in (22) for microscopic system (i.e., $10^{-6}<\alpha<10^{-1}$ and $\gamma_{D} \approx 10^{2}$ at $\left.T=1^{\circ} \mathrm{K}\right)$ [36], gives:

$10^{-14} s<\frac{1}{\kappa} \approx \frac{2 \pi}{\omega}<10^{-9} s$

and

$10^{-12} \frac{\mathrm{m}^{2}}{\mathrm{~s}}<D<10^{-2} \frac{\mathrm{m}^{2}}{\mathrm{~s}}$

\section{INTEGRATION OF THE QUANTUM-STOCHASTIC MOTION EQUATION}

The Markov process (21) for $V_{q u}=V_{q u(q)}$ not function of time (e.g., $V_{q u}=$ constant $=0$ ), obeys the Smolukowski integro-differential equation for the Markov probability transition function (PTF) [46]:

$P\left(q, q_{0} \mid t+\tau, t_{0}\right)=\int_{-\infty}^{\infty} P(q, z \mid \tau, t) P\left(z, q_{0} \mid t-t_{0}, t_{0}\right) d^{r} z$

where the $\operatorname{PTF} P(q, z \mid \tau, t)$ represents the probability that a quantity of PMD $n(q, t)$ at instant $\mathrm{t}$, in a time interval $\tau$, in a point $\mathrm{z}$, is transferred to the point $q$ [46].

The conservation of the PMD $n$ in integral form shows that the PTF generates the displacement of a vector $(q, t)-(z, 0)$ according to the rule [46]:

$n(q, t)=\int P(q, z \mid t, 0) n_{(z, 0)} d^{r} z$

\section{A. The Simplest Case: The Stationary Quasi-eigenstates}

Generally speaking, for the quantum case, equation (21) is not described by a Fokker-Planck equation (FPE), since the quantum potential $V_{q u_{(n)}}$ owns a functional dependence by $n(q, t)$ and the PTF $P\left(q, q_{0} \mid t+\tau, t_{0}\right)$ is non-Gaussian.

Nevertheless, if the initial distribution $n_{\left(q, t_{0}\right)}$ is stationary (e.g., of a quantum eigenstate [36]) and is close to the longtime final stationary distribution of the stochastic case (i.e., $\left.n_{\left(q, t_{0}\right)} \approx n_{e q(q, t=\infty)}\right)$ condition that can be checked at the end, it is possible to assume the approximation:

$$
V_{q u} \cong-\left(\frac{\hbar^{2}}{4 m}\right)\left(\left(\frac{\partial^{2} \ln n_{e q(q)}}{\partial q^{2}}\right)+\frac{1}{2}\left(\frac{\partial \ln n_{e q(q)}}{\partial q}\right)^{2}\right)
$$

so that the PMD $n$ approaches the limiting behavior given by the Fokker-Plank equation:

$\frac{\partial P_{(q, z \mid t, 0)}}{\partial t}+\frac{\partial P_{(q, z \mid t, 0)}}{\partial q}=0$

where

$v=-\frac{1}{m \kappa} \frac{\partial\left(V_{(q)}-\left(\frac{\hbar^{2}}{4 m}\right)\left(\left(\frac{\partial^{2} \ln n}{\partial q^{2}}\right)+\frac{1}{2}\left(\frac{\partial \ln n}{\partial q}\right)^{2}\right)\right)}{\partial q}-\frac{D}{2} \frac{\partial \ln n}{\partial q}$

leading to the final equilibrium identity 


$$
\begin{aligned}
& \frac{1}{m \kappa} \frac{\partial\left(V_{(q)}-\left(\frac{\hbar^{2}}{4 m}\right)\left(\left(\frac{\partial^{2} \ln n_{e q}}{\partial q^{2}}\right)+\frac{1}{2}\left(\frac{\partial \ln n_{e q}}{\partial q}\right)^{2}\right)\right)}{\partial q}+\frac{D}{2} \frac{\partial \ln n_{e q}}{\partial q} \equiv \operatorname{ligm}_{\Delta t \rightarrow 0}(4 \pi D \Delta t)^{-1 / 2} e^{-\frac{\Delta w_{k}^{2}}{4 \Delta t}} \lim _{\Delta t \rightarrow 0} \mathscr{P}\left(\Delta \mathcal{W}_{k}, \Delta t\right) \\
& \text { In appendix B equation (30) is used to derive the stationary } \quad=\lim _{\Delta t \rightarrow 0}(4 \pi D \Delta t)^{-1 / 2} e^{-\frac{1}{4 \Delta t} \frac{\left(q_{k+1}-<q_{k+1}>\right)^{2}}{D}} \\
& \text { state of linear systems in presence of small noise. } \\
& \text { B. Evolution of Quantum Superposition of States }=(4 \pi D \Delta t)^{-1 / 2} e^{-\frac{1}{4 \Delta t} \frac{\left(q_{k+1}-q_{k}-<\dot{\bar{q}}_{k}>\Delta t-\frac{<\ddot{\bar{q}}_{k}>}{2} \Delta t^{2}\right)^{2}}{D}} \\
& \text { Submitted to Fluctuations }
\end{aligned}
$$

\section{B. Evolution of Quantum Superposition of States Submitted to Fluctuations}

In order to determine the evolution of quantum superposition of states, that are not stationary, we need to integrate the stochastic differential equation (SDE).

$$
\begin{aligned}
\dot{q}=-\gamma_{D} \frac{\mathcal{L}^{2}}{8 \alpha \hbar} & \frac{\partial\left(V_{(q)}-\left(\frac{\hbar^{2}}{2 m}\right)\left(\left(\frac{\partial^{2} \ln n}{\partial q^{2}}\right)+\left(\frac{\partial \ln n}{\partial q}\right)^{2}\right)\right)}{\partial q} \\
& +\sqrt{\gamma_{D}} \frac{\mathcal{L}}{2} \sqrt{\frac{k T}{\hbar}} \xi_{(t)}
\end{aligned}
$$

As shown below, this can be done by using the discrete approach with the help of the Smolukowski integrodifferential equation (25) and the associated conservation equation (26) for the PMD.

Given the presence of the quantum pseudo-potential, this procedure is not general and holds under some approximated conditions, introduced further on in the derivation.

We integrate the SDE (31) by using its $2^{\text {nd }}$ order discrete expansion:

$$
\begin{aligned}
q_{k+1} \cong & q_{k}-\frac{1}{m \kappa} \frac{\partial\left(V_{\left(q_{k}\right)}+V_{q u\left(n_{q_{k}}, t_{k}\right)}\right)}{\partial q_{k}} \Delta t_{k} \\
& -\frac{1}{m \kappa} \frac{d}{d t} \frac{\partial\left(V_{\left(q_{k}\right)}+V_{q u\left(n_{q_{k}}, t_{k}\right)}\right)}{\partial q_{k}} \frac{\Delta t_{k}{ }^{2}}{2}+D^{1 / 2} \Delta \mathcal{W}_{k}
\end{aligned}
$$

where

$$
\begin{aligned}
& q_{k}=q_{\left(t_{k}\right)} \\
& \Delta t_{k}=t_{k+1}-t_{k} \\
& \Delta \mathcal{W}_{k}=\mathcal{W}_{\left(t_{k+1}\right)}-\mathcal{W}_{\left(t_{k}\right)}
\end{aligned}
$$

where the PMD $n\left(q_{k}, t_{k}\right)$ from (26) reads:

$n\left(q_{k}, t_{k}\right)=\int_{-\infty}^{\infty} P\left(q_{k}, q_{0} \mid t_{k}, 0\right) n\left(q_{0}, 0\right) d q_{0}$

where $\Delta \mathcal{W}_{k}$ has Gaussian zero mean and unitary variance whose probability function $\mathscr{P}\left(\Delta \mathcal{W}_{k}, \Delta t\right)$, for $\Delta t_{k}=\Delta t \forall k$, reads: where

$$
\begin{aligned}
<q_{k+1}>\cong & <q_{k}>-<\frac{1}{m \kappa} \frac{\partial\left(V_{\left(q_{k}\right)}+V_{q u\left(n_{q_{k}}\right)}\right)}{\partial q_{k}}>\Delta t_{k} \\
& -<\frac{1}{m \kappa} \frac{d}{d t} \frac{\partial\left(V_{\left(q_{k}\right)}+V_{q u\left(n_{q_{k}}\right)}\right)}{\partial q_{k}}>\frac{\Delta t_{k}^{2}}{2}
\end{aligned}
$$

where it has been introduced the midpoint approximation:

$\bar{q}_{k}=\frac{q_{k+1}+q_{k}}{2}$

and where

$<\dot{\bar{q}}_{k}>=-\frac{1}{m \kappa} \frac{\partial\left(V_{\left(\bar{q}_{k}\right)}+V_{q u\left(n_{\bar{q}_{k}}, t_{k}\right)}\right)}{\partial \bar{q}_{k}}$

and

$<\ddot{\bar{q}}_{k}>=-\frac{1}{2 m \kappa} \frac{d}{d t} \frac{\partial\left(V_{\left(\bar{q}_{k}\right)}+V_{q u\left(n_{\bar{q}_{k}}, t_{k}\right)}\right)}{\partial \bar{q}_{k}}$

By using standard manipulations [45], from (37), the PTF reads [36]:

$P\left(q, q_{0}, t, t_{0}\right)=\lim _{\Delta t \rightarrow 0} P\left(q_{n}, q_{0} \mid n \Delta t, 0\right)$

$=\lim _{\Delta t \rightarrow 0} \int_{-\infty}^{\infty} \prod_{k=1}^{n} d q_{k-1} \mathscr{P}\left(q_{k}, q_{k-1} \mid \Delta t,(k-1) \Delta t\right)$

$=\left(\exp \int_{q_{0}}^{q} \frac{1}{2 D}<\dot{q}>_{(q, t)} d q\right)$

$\int_{q_{0}}^{q} \mathcal{D} q \exp -\frac{1}{4 D} \int_{t_{0}}^{t} d t\left(\dot{q}^{2}+<\dot{q}>^{2}+2 D \frac{\partial<\dot{q}>}{\partial q}\right)$

where the discrete PTF $\mathscr{P}\left(q_{k}, q_{k-1} \mid \Delta t,(k-1) \Delta t\right)$ reads: 
$\mathbb{P}\left(q_{k}, q_{k-1} \mid \Delta t,(k-1) \Delta t\right)$

$\left.\begin{array}{l}=(4 \pi D \Delta t)^{-1 / 2} \exp \frac{\Delta t}{2 D}\left[\begin{array}{l}-<\dot{\bar{q}}_{k-1}>\left(\frac{q_{k}-q_{k-1}}{\Delta t}\right) \\ -\frac{1}{2}\left(\frac{q_{k}-q_{k-1}}{\Delta t}\right)^{2}+<\dot{\bar{q}}_{k-1}>^{2} \\ -2 D \frac{\partial<\dot{\bar{q}}_{k-1}>}{\partial \bar{q}_{k-1}}\end{array}\right]\end{array}\right]$.

Before proceeding further, we observe that, generally speaking, since the quantum potential is a function of the PMD $n\left(q_{k}, t_{k}\right)$, the evolution of equation (32) depends on the exact sequence of the noise inputs $D^{1 / 2} \Delta \mathcal{W}_{k}$ and, therefore, also on the discrete time interval of integration. This behavior can be easily verified by performing the numerical integration of (32). The vagueness of the problem can be analytically identified by the fact that, in equation (43), $<\dot{\bar{q}}_{k-1}>$ and $\frac{\partial<\dot{\bar{q}}_{k-1}>}{\partial q_{k-1}}$ depend on $n\left(q_{k}, k \Delta t\right)$ and $n\left(q_{k+1},(k+1) \Delta t\right)$ that define the quantum potential values $V_{q u_{\left(n_{k}\right)}}, V_{q u_{\left(n_{k+1}\right)}}$ that are unknown at the moment $(k-1) \Delta t$.

Although there is no general solution to this problem, in the limit of small speed $\dot{q}_{k}$ and small noise amplitude [35], [36], [42] it is possible to proceed by successive steps of approximation if, for the discrete time interval $\Delta t$, the speed change is small enough to have (at the zero order of approximation) that:

$$
\frac{<\dot{q}_{k+1}>}{<\dot{q}_{k}>}=1+<\varepsilon>
$$

with $<\varepsilon>\ll 1$, and that:

$<\dot{\bar{q}}_{k}>=\frac{<\dot{q}_{k+1}>+<\dot{q}_{k}>}{2}=<\dot{q}_{k}>\left(1+\frac{<\varepsilon>}{2}\right) \cong<\dot{q}_{k}>$

The condition $\lim _{\Delta t \rightarrow 0} \lim _{D \rightarrow 0}<\varepsilon>\rightarrow 0$ is guaranteed by the existence of the deterministic limit (see appendix C) that implies that:

$\lim _{\Delta t \rightarrow 0} \lim _{D \rightarrow 0} \dot{q}_{k}=\lim _{\Delta t \rightarrow 0}<\dot{\bar{q}}_{k}>=<\dot{q}_{k}>$

Therefore, given the discrete time interval $\Delta t$, there exists a sufficiently small noise amplitude, as well as small diffusion coefficient $D=\gamma_{D} \frac{\mathcal{L}^{2}}{4} \frac{k T}{\hbar}$, to proceed by successive steps of approximation from the zero-order PTF (see Appendix B):
$\mathbb{P}^{(0)}\left(q_{k}, q_{k-1} \mid \Delta t,(k-1) \Delta t\right)$

$=(4 \pi D \Delta t)^{-1 / 2} e^{\frac{\Delta t}{4 D}\left[\begin{array}{l}-\left(\dot{q}_{k-1}-\frac{<\dot{q}_{k}>+<\dot{q}_{k-1}>}{2}\right)^{2} \\ +D \frac{\partial<\dot{q}_{k}>+<\dot{q}_{k-1}>}{\partial \bar{q}_{k-1}}\end{array}\right]}$

$=(4 \pi D \Delta t)^{-1 / 2} e^{\frac{\Delta t}{4 D}\left[\begin{array}{l}-\left(\dot{q}_{k-1}-<\dot{q}_{k-1}>\left(1+\frac{<\varepsilon>}{2}\right)\right)^{2} \\ +D \frac{\partial<\dot{q}_{k}>+<\dot{q}_{k-1}>}{\partial \bar{q}_{k-1}}\end{array}\right]}$

$\cong(4 \pi D \Delta t)^{-1 / 2} e^{\frac{\Delta t}{4 D}\left[-\left(\dot{q}_{k-1}-<\dot{q}_{k-1}>\right)^{2}+2 D \frac{\partial<\dot{q}_{k-1}>}{\partial q_{k-1}}\right]}$

which can be used to find the zero order of approximation of the PMD in the next instant of time

$$
n^{(0)}\left(q_{k}, k \Delta t\right)=\int_{-\infty}^{\infty} \mathcal{P}^{(0)}\left(q_{k}, q_{k-1 \mid \Delta t,(k-1) \Delta t)} n_{\left(q_{k-1,(k-1) \Delta t}\right)} d q_{k-1}\right.
$$

and find the approximate zero-order values of the quantum potential at the next instant $\mathrm{k}$ for calculating:

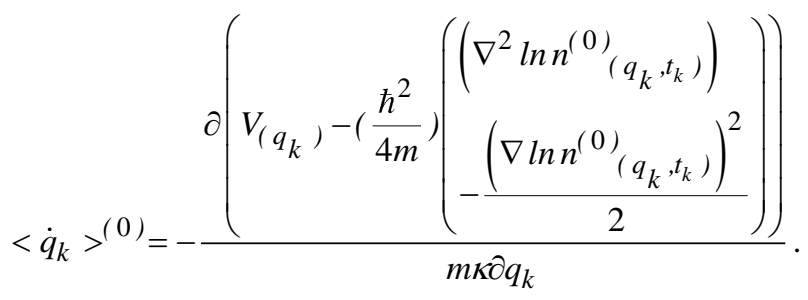

Thus, (48)-(49) allows us to obtain, in the next approximation order, a more accurate PTF that reads:

$\mathbb{P}^{(1)}\left(q_{k}, q_{k-1} \mid \Delta t,(k-1) \Delta t\right)$

$=(4 \pi D \Delta t)^{-1 / 2} \exp \frac{\Delta t}{4 D}\left[\begin{array}{l}-\left(\dot{q}_{k-1}-\frac{<\dot{q}_{k}>^{(0)}+<\dot{q}_{k-1}>}{2}\right)^{2} \\ +D \frac{\partial<\dot{q}_{k}>^{(0)}+<\dot{q}_{k-1}>}{\partial \bar{q}_{k-1}}\end{array}\right]$

$\cong(4 \pi D \Delta t)^{-1 / 2} \exp \frac{\Delta t}{4 D}\left[\begin{array}{l}-\left(\dot{q}_{k-1}-\frac{<\dot{q}_{k}>^{(0)}+<\dot{q}_{k-1}>}{2}\right)^{2} \\ +2 D\left(\frac{\partial<\dot{q}_{k}>^{(0)}}{\partial q_{k}}+\frac{\partial<\dot{q}_{k-1}>}{\partial q_{k-1}}\right)\end{array}\right]$

as well as the associated PDF

$\left.n_{\left(q_{k}, k \Delta t\right)}^{(1)}=\int_{-\infty}^{\infty} \mathscr{P}^{(1)}\left(q_{k}, q_{k-1} \mid \Delta t,(k-1) \Delta t\right) n_{\left(q_{k-1},(k-1)\right.} \Delta t\right) d q_{k}$ 
and the mean velocity

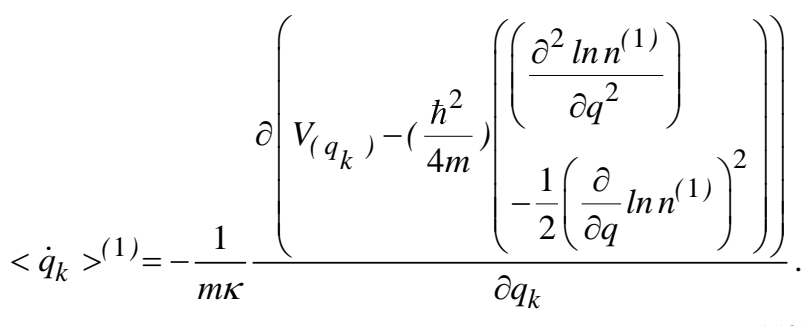

Thence, repeating the procedure, at successive $u$-th order of approximation $(u=2,, 3, \ldots \ldots . . r)$ we obtain:

$$
\begin{aligned}
& \mathbb{P}^{(u)}\left(q_{k}, q_{k-1} \mid \Delta t,(k-1) \Delta t\right) \\
& =(4 \pi D \Delta t)^{-1 / 2} \exp \frac{\Delta t}{4 D}\left[\begin{array}{l}
-\left(\dot{q}_{k-1}-\frac{<\dot{q}_{k}>^{(u-1)}+<\dot{q}_{k-1}>}{2}\right)^{2} \\
+D \frac{\partial<\dot{q}_{k}>^{(u-1)}+<\dot{q}_{k-1}>}{\partial \bar{q}_{k-1}}
\end{array}\right] \\
& \cong(4 \pi D \Delta t)^{-1 / 2} \exp \frac{\Delta t}{4 D}\left[\begin{array}{l}
-\left(\dot{q}_{k-1}-\frac{\left\langle\dot{q}_{k}>^{(u-1)}+<\dot{q}_{k-1}>\right.}{2}\right)^{2} \\
+D\left(\frac{\partial<\dot{q}_{k}>^{(u-1)}}{\partial q_{k}}+\frac{\partial<\dot{q}_{k-1}>}{\partial q_{k-1}}\right)
\end{array}\right]
\end{aligned}
$$

and

$$
n^{(u)}{ }_{\left(q_{k}, k \Delta t\right)}=\int_{-\infty}^{\infty} \mathscr{P}^{(u)}{ }_{\left(q_{k}, q_{k-1} \mid \Delta t,(k-1) \Delta t\right)} n_{\left(q_{k-1},(k-1) \Delta t\right)} d q_{k}
$$

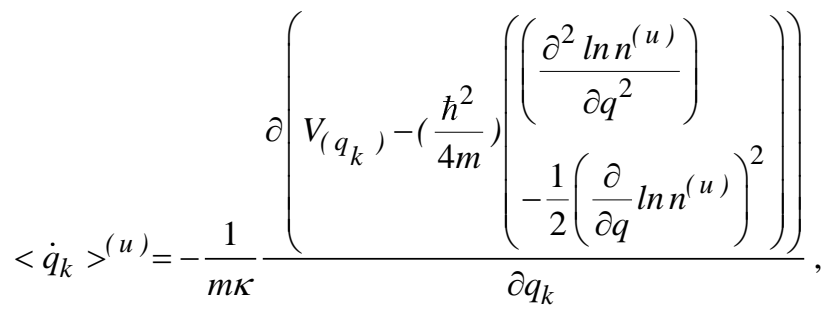

so that the final PTF $\mathscr{P}\left(q_{k}, q_{k-1} \mid \Delta t,(k-1) \Delta t\right)$ can be obtained by the convergence of the limit:

$\mathbb{P}^{(\infty)}\left(q_{k}, q_{k-1} \mid \Delta t,(k-1) \Delta t\right)$

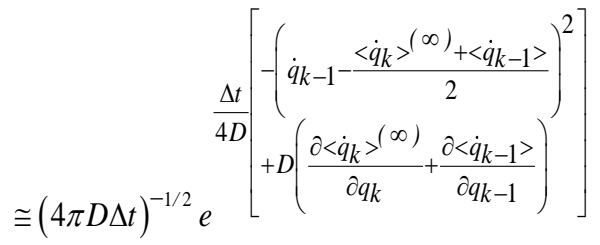

Finally, by using $\mathscr{P}^{(\infty)}\left(q_{k}, q_{k-1} \mid \Delta t,(k-1) \Delta t\right)$, the PMD at the $k$-th instant reads:

$$
n_{\left(q_{k}, k \Delta t\right)}^{(\infty)}=\int_{-\infty}^{\infty} \mathscr{P}_{\left(q_{k}, q_{k-1} \mid \Delta t,(k-1) \Delta t\right)} n_{\left(q_{k-1},(k-1) \Delta t\right)}^{(\infty)} d q_{k-1}
$$

leading to the velocity field

$$
<\dot{q}_{k}>^{(\infty)}=-\frac{1}{m \kappa} \frac{\partial\left(V_{\left(q_{k}\right)}-\left(\frac{\hbar^{2}}{4 m}\right)\left(\begin{array}{l}
\left(\frac{\partial^{2} \ln n^{(\infty)}}{\partial q^{2}}\right) \\
\left.\left.-\frac{1}{2}\left(\frac{\partial}{\partial q} \ln n^{(\infty)}\right)^{2}\right)\right)
\end{array}\right)\right.}{\partial q_{k}}
$$

and to the continuous PTF solution

$$
\begin{aligned}
& P\left(q, q_{0} \mid t-t_{0}, 0\right)=\lim _{\Delta t \rightarrow 0} P^{(\infty)}\left(q_{n}, q_{0} \mid n \Delta t, 0\right) \\
& =\lim _{\Delta t \rightarrow 0} \int_{-\infty}^{\infty} \prod_{k=1}^{n} d q_{k-1} \mathscr{P}^{(\infty)}\left(q_{k}, q_{k-1} \mid \Delta t,(k-1) \Delta t\right) \\
& \left.=\int_{q_{0}}^{q} \mathscr{D} q e^{\left[-\frac{1}{2 D} \sum_{k=1}^{n}<\dot{\bar{q}}_{k-1}>^{(\infty)} \Delta q_{k}\right.}\right]_{e}-\frac{\Delta t}{4 D} \sum_{k=1}^{n}\left(\begin{array}{l}
\left(\frac{q_{k}-q_{k-1}}{\Delta t}\right)^{2} \\
+<\dot{\bar{q}}_{k-1}>^{(\infty)^{2}} \\
-2 D \frac{\partial<\overline{\bar{q}}_{k-1}>^{(\infty)}}{\partial \bar{q}_{k-1}}
\end{array}\right)
\end{aligned}
$$

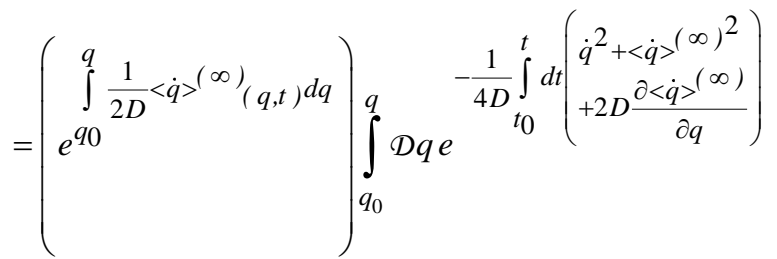

where

$<\dot{\bar{q}}_{k-1}>^{(\infty)}=\frac{<\dot{q}_{k}>^{(\infty)}+<\dot{q}_{k-1}>}{2}$.

The general solution, given in the recursion formula (59), can be applied also to non-linear system that cannot be treated by standard approaches [15].

\section{General Characteristics of the quantum Relaxation}

For potentials not function of time, the Brownian process described by the FPE admits the stationary long-time solution

$$
\begin{aligned}
& P\left(q, q_{-\infty} \mid t-t_{-\infty}, t_{-\infty}\right) \\
& =\lim _{t_{0} \rightarrow-\infty} N \exp \frac{1}{D} \int_{q_{-\infty}}^{q}\left\langle\dot{q}>_{\left(q^{\prime}\left(t, t_{0}\right)\right.}\right) d q^{\prime}
\end{aligned}
$$

$=N \exp \frac{1}{D} \int_{q_{-\infty}}^{q} K\left(q^{\prime}\right) d q^{\prime}$ 
where

$K(q)=-\frac{1}{m \kappa} \frac{\partial V_{(q)}}{\partial q}$

leading to the canonical expression [45]:

$$
\begin{aligned}
& P\left(q, q_{0} \mid t-t_{0}, t_{0}\right) \\
& =\left(e^{\int^{q_{0}} \frac{1}{2 D} K\left(q^{\prime}\right) d q^{\prime}}\right) \int_{q_{0}}^{q} D e^{-\frac{1}{4 D} \int_{t_{0}}^{t} d t\left(\dot{q}^{2}+K^{2}(q)+2 D \frac{\partial K(q)}{\partial q}\right)}
\end{aligned}
$$

Generally speaking, in the quantum case (59), $\left\langle\dot{q}>^{(\infty)}{ }_{(q, t)}\right.$ cannot be given in a closed form since it depends on the specific relaxation path $n_{(q, t)}$ of the system toward the steady state which significantly depends on the initial conditions $\left.n_{\left(q, t_{0}\right)},<\dot{q}\right\rangle_{\left(q, t_{0}\right)}$ and, therefore, on the initial time $t_{0}$

Thus, in principle, different long-period stationary configurations $n_{(q, t=\infty)}$ can be reached, depending on the initial time $t_{0}$ in which the quantum superposition of states is submitted to fluctuations.

Furthermore, since the PMD provides information about the MDD $\tilde{n}_{(q, t)}$ only at leading order of approximation of $\dot{q}$ (i.e., slow relaxation process, and small amplitude of fluctuations [42]), in the case of a large fluctuation (that can occur on a large time scale), $\tilde{n}_{(q, t)}$ can make a transition, not described by (59) (even for instance from a stationary eigenstate to a generic superposition of states) possibly leading to a new relaxation toward a different stationary eigenstate.

Due to this jumping process of large fluctuations, the PMD $n_{(q, t)}$ can only describe the relaxation process after each

large fluctuation, but not the complete evolution of the system. In a collection of a large number of particles, while on short time scale each one relaxes up to an eigenstate, on long time scale the overall system relaxes toward the statistical mixture.

As a function of the temperature dependence of the diffusion coefficient $D$, the corresponding statistical distribution is determined.

\section{DISCUSSION}

In order to critically examine the SQHM, it is worth mentioning that equations (1)-(3) constitute an approximated version [42].

Approximation (7) leads to the Langevin-Schrodinger equation and, under certain conditions, to the macroscopic classical mechanics.

The constancy of $\kappa$ is an "ad hoc" assumption but, generally speaking, it is not [42], [46], [47]. This approximation is mainly due to obtaining a manageable model able of capturing the characteristics of the quantum evolution in presence of noise.

Furthermore, the dissipation parameter $\alpha$, which in principle should be self-defined, is introduced semiempirically. The assumption of its smallness is justified for microscopic systems close to the "deterministic" quantum limit (see condition (16)) but its order of magnitude for a physical system is not known and must be measured.

Finally, it should be noted that the hypothesis that the evolution of the PMD $n$ is physically significant (being related to that of the real MDD $\tilde{n}$ in the low amplitude limit of noise) is justified by the condition $\lim _{T \rightarrow 0} n=\lim _{T \rightarrow 0} \tilde{n}=|\psi|^{2}$ but, in general, the basin of convergence of this limit is not defined.

\section{A. The Emerging of Classical Behavior on Coarse- grained Large-scale Description}

Is matter of fact that, if the quantum potential is cancelled by hand in the quantum hydrodynamic equations (1)-(3), the classical equation of motion emerges [29], [30]. Even if this is true, this operation is not mathematically correct since it changes the characteristics of the Madelung's equations [33]. Doing so, the stationary configurations (i.e., eigenstates) are wiped out because we cancel the balancing of the quantum potential against the Hamiltonian forces. Thus, an even small quantum potential cannot be neglected in the deterministic Madelung's model.

Moreover, even if the noise has zero mean, the average of the quantum potential fluctuations $\bar{V}_{s t(n, S)} \cong \beta S$, is not zero so that the effect on $\dot{q}$ and $\ddot{q}$ is not equal (as a mean) to that of the deterministic quantum potential. Thus, the stochastic sequence of inputs of noise alters the coherent reconstruction of superposition of state.

In agreement with the decoherence theory [46], the SQHM shows that this phenomenon is associated with the appearance the dissipative force (e.g., $-\kappa \dot{q}_{(t)}$ in (2)).

If on systems with physical length smaller than the De Broglie length $\lambda_{c}$ the fluctuations amplitude are progressively cancelled, on the contrary, if the physical scale is much larger than $\lambda_{c}$, the non-local force, generated by the quantum potential, can be much smaller that the fluctuations amplitude so that:

$\lim _{q \rightarrow \Delta q \gg \lambda_{c}} \frac{1}{m}\left|\frac{\partial V_{q u(n)}}{\partial q_{i}}\right| \ll \kappa\left(\frac{\mathcal{L}}{\lambda_{c}}\right)\left(\frac{\hbar}{2 m}\right)^{\frac{1}{2}}=\kappa \frac{\mathcal{L}}{2} \sqrt{\frac{k T}{2 \hbar}}$,

and can be disregarded (noise shielding).

Therefore, in the coarse-grained description of a macroscopic system whose resolution size $\Delta q$ (e.g., the side of the elemental cube of volume) is larger than the quantum potential range of interaction (see below (68)) [42], the classical stochastic behavior can emerge.

Actually, in order to have a large-scale description, completely free from quantum potential interaction, we can more strictly require 
$\lim _{\frac{q}{\lambda_{c}} \rightarrow \infty}\left|\frac{\partial V_{q u(n(q))}}{\partial q_{i}}\right|=\lim _{\frac{q}{\lambda_{c}} \rightarrow \infty} \sqrt{\frac{\partial V_{q u(n(q))}}{\partial q_{i}} \frac{\partial V_{q u(n(q))}}{\partial q_{i}}}=0$

For instance, systems that interact by Lennard-Jones potential for which the long-distance wave function reads [47]:

$$
\lim _{q \rightarrow \infty}|\psi| \propto a^{-1 / 2} \frac{1}{q}
$$

the quantum potential at large distance reads:

$$
\begin{aligned}
& \lim _{q \rightarrow \infty} \frac{\partial V_{q u(n)}}{\partial q}=\lim _{q \rightarrow \infty} \frac{\hbar^{2}}{2 m} \frac{\partial}{\partial q} \frac{1}{|\psi|} \frac{\partial^{2}|\psi|}{\partial q \partial q} \\
& \approx \frac{\hbar^{2}}{2 m} \frac{\partial}{\partial q} q \frac{\partial^{2} \frac{1}{q}}{\partial q \partial q} \approx-2 \frac{\hbar^{2}}{m} \frac{1}{q^{3}}
\end{aligned}
$$

and the large-scale classical behavior may appear [35], [48] in a sufficiently rarefied phase (where the mean inter-particle distance is much larger than the quantum potential range of interaction).

By observing that in order to fulfill the condition (64), it is enough to have:

$$
\int_{0}^{\infty} r^{-1}\left|\frac{1}{m} \frac{\left.\partial V_{q u\left(n_{(} q_{i}\right)}\right)}{\partial q_{i}}\right|_{(r, \theta, \varphi)} d r=\text { limited } \forall \theta, \varphi,
$$

It is possible to define the measure of the length of interaction of the quantum potential that, for $m \neq 0$, reads [35]:

$$
\lambda_{q u}=\lambda_{c} \frac{\int_{0}^{\infty} r^{-1}\left|\frac{\left.\partial V_{q u\left(n_{(q}\right)}\right)}{\partial q_{i}}\right|_{(r, \theta, \varphi)} d r}{\left|\frac{\left.\partial V_{q u\left(n_{(q}\right)}\right)}{\partial q_{i}}\right|_{\left(r=\lambda_{c}, \theta, \varphi\right)}} .
$$

Thus, since for linear systems [42]:

$$
\frac{\partial V_{q u(n)}}{\partial q_{i}} \propto q
$$

the length of quantum potential interaction (i.e., the quantum entanglement distance) results:

$$
\lambda_{q u} \propto \int_{0}^{\infty} d q=\infty
$$

and therefore, quantum effects can appear even in a largescale coarse-grained description.
For stronger that linearly bonded systems (i.e., $\left.\lim _{q \rightarrow \infty} V_{(q)} \propto q^{n>2}\right)$, due to the higher localization of the wave function [35], the quantum potential for the eigenstates at large distance grows faster (i.e., $\lim _{q \rightarrow \infty} V_{q u(q)} \propto q^{m}$ where $m>2$ ) and therefore $\lambda_{q u}=\infty$ too, with quantum phenomena that also have a macroscopic manifestation [35], [48].

On the contrary for a system of Lennard-Jones interacting particles the quantum entanglement distance reads:

$\lambda_{q u} \cong \int_{0}^{d} d q+\lambda_{c}^{4} \int_{d}^{\infty} \frac{1}{q^{4}} d q=d+\frac{\lambda_{c}}{3}\left(\frac{\lambda_{c}}{d}\right)^{3}$

where $d=r_{0}(1+\varepsilon)$ is the distance up to which the interatomic force is approximately linear (where $r_{0}$ is the atomic equilibrium distance).

An experimental confirmation of the physical relevance of quantum entanglement length can come from the quantum to classical transition that happens in crystalline solid at melting point when the system passes from a quantum lattice to a fluid amorphous classical phase $\left(\lambda_{c} \approx r_{0}\right)$ [35].

Assuming that in the quantum lattice the atomic wavefunction spans itself not further than the quantum entanglement distance, it follows that at the melting point its variance equals $\lambda_{q u}-r_{0}$.

On these assumptions, the theoretical value of the Lindemann constant (i.e., the wave function variance at the melting point) is related to $\lambda_{q u}$ that reads:

$\lambda_{q u} \approx r_{0}\left((1+\varepsilon)+\frac{1}{3}\left(\frac{1}{(1+\varepsilon)}\right)^{3}\right) \approx r_{0}\left(1+\frac{1+2 \varepsilon}{3}\right)$

where being $\varepsilon \approx 0,05 \div 0,1$, leads to:

$\lambda_{q u} \approx(1,36 \div 1,39) r_{0}$.

More precise evaluation, making use of the potential well model for the molecular interaction, leads to $\lambda_{q u} \cong 1,2357 r_{0}$

[35], that gives the value of the Lindemann constant of 0,2357 that well agrees with the experimental measurements ranging between 0,2 and 0,25 [35].

Also, the $\mathrm{He}_{4}$ fluid-superfluid transition can be described by introducing the finite range of quantum interaction in the intermolecular potential as a function of temperature [35], [48].

In SQHM, macroscopicity is introduced into the theory by the intrinsic presence of the De Broglie physical length while the transition from quantum stochastic to classically stochastic dynamics is determined by the distance of the quantum entanglement (i.e., $\lambda_{q u}$ ). Thus, for $\lambda_{q u} \gg \lambda_{c}$ macroscopic quantum phenomena [35], [48] are also possible. 


\section{1) From Micro to Macro Description}

Given the PMD current $J_{j(q, t)}=n \dot{q}_{j_{(t)}}$, that reads:

$J_{(q, t)}=n \dot{q}_{(t)}=-n\left(\frac{m \mathcal{L}^{2}}{4 \alpha} \frac{\partial\left(V_{(q)}+V_{q u}\right)}{\partial q}+\varpi_{(q, t, T)}\right)$

The macroscopic behavior can be obtained by the discrete coarse-grained spatial description of (74) as a function of the local $j$-th cell of side $l$, that reads [45]:

$$
\begin{aligned}
d x_{j}= & -\frac{m \mathcal{L}^{2}}{4 \alpha} \mathscr{D}_{j m}^{\prime} x_{(m)}\left(\mathcal{D}_{m k} V_{k}+\mathscr{D}_{m k}^{q u} V_{q u_{k}}\right) d t \\
& +\mathscr{D}^{\prime \prime}{ }_{j k} x_{(k)} \Phi_{(k)} d W_{k(t)}
\end{aligned}
$$

where

$$
\begin{aligned}
& x_{j}=l^{3} n_{\left(q_{j}, t\right)}, \\
& V_{k}=V_{\left(q_{k}\right)}, \\
& V_{q u_{k}}=V_{q u\left(n_{\left(q_{k}\right)}\right)}, \\
& \Phi_{k}=\Phi_{\left(q_{k}, t\right)},
\end{aligned}
$$

where

$$
\lim _{l \rightarrow 0} l^{-6}<\Phi_{j}, \Phi_{k}>=<\varpi_{\left(q_{j}\right)}, \varpi_{\left(q_{k}\right)}>_{(T)} F_{(l(k-j))} .
$$

and where the terms $\mathscr{D}_{j k}, \mathscr{D}^{\prime}{ }_{j k}, \mathscr{D}^{\prime \prime}{ }_{j k}$ and $\mathscr{D}_{m k}^{q u}$ are matrices of coefficients corresponding to the discrete approximation of the derivatives $\frac{\partial}{\partial q_{k}}$ at the $j$-th point.

Generally speaking, the quantum potential interaction $V_{q u_{k}}$ stemming by the $k$-th cell, depends by the strength of the Hamiltonian potential $V_{\left(q_{k}\right)}$.

By setting, in a system of a huge number of particles, the side length $\mathcal{L}$ equal to the mean intermolecular distance $\mathcal{L}$, we can have a classical rarefied phase, if $\mathcal{L}$ is much bigger than the quantum entanglement length $\lambda_{q u}$ (that by (68)) is also a function of the De Broglie length).

Typically, given that for the Lennard-Jones potential:

$$
\begin{aligned}
& \lim _{q \rightarrow \infty}-\frac{\partial V_{q u(n)}}{\partial q} \approx 2 \frac{\hbar^{2}}{m \lambda_{c}^{3}} \frac{1}{\left(\frac{q}{\lambda_{c}}\right)^{3}}, \\
& \approx \frac{k T}{\hbar} \sqrt{\frac{m k T}{2}} \frac{1}{\left(\frac{q}{\lambda_{c}}\right)^{3}}=0
\end{aligned}
$$

the quantum potential range of interaction $\lambda_{q u}$ is finite, for $\mathcal{L} \gg \lambda_{q u}$ the contribution to the $j$-th cell from the $k$-th is null for $k \neq j$ and $\mathscr{D}_{m k}^{q u}$ is diagonal. Thus, the quantum effects are confined to each single molecular domain.

Furthermore, being $\mathcal{L} \gg \lambda_{c} \cup \lambda_{q u}$ it follows that the spatial correlation length of the noise results $F_{(k-j)} \propto \delta_{k j}$ and it follows that the fluctuations appears spatially uncorrelated in macroscopic systems

On the contrary, given that for stronger than linearly interacting systems $\lambda_{q u} \rightarrow \infty$, the contribution to the $j$-th cell from the $k$-th is not null; and the quantum interaction (i.e., the quantum entanglement) appears on the coarse-grained description. Actually, by using the SQHM it is possible to derive descriptions for dense phases where quantum effects appear on macroscopic scale [35], [48].

Furthermore, it is worth mentioning that the physical possibility to have macroscopic systems with quantum characters, in the case of highly condensed system, can have important consequence also in the cosmological physics. In fact, since the repulsive force generated by the quantum potential in the collapsed mass of black holes becomes very high, it can possibly stop its collapse and remove the classical singularity [49] with the establishment of stationary mass distributions (i.e., quantum eigenstates).

\section{B. Minimum Uncertainty in the SQHM}

The quantum theory, that describes the physical evolution as a function both of the interactions strength and the lengthscale of the system, must show a connection between the properties of the quantum mechanics and the large-scale classical limit.

The most characterizing laws of the two descriptions are the minimum uncertainty of quantum mechanics and the finite speed of propagation of interactions and information of the local classical relativity.

If on distances $\mathcal{L}_{q}$ smaller than $\lambda_{c}$ any system fully approaches the "deterministic" quantum mechanics so that its subparts are not independent each other, it follows that, in order to assume information from the observed system, it is necessary that the observer must be far apart (before and after the process) more than the distance $\mathcal{L}_{q}$ (see Appendix D) and hence, for the finite speed of propagation of interactions and information, information cannot be acquired in a time shorter than:

$\Delta \tau_{\text {min }}=\frac{\mathcal{L}_{q}}{c} \propto \frac{\lambda_{c}}{c} \propto \frac{2 \hbar}{\left(2 m c^{2} k T\right)^{1 / 2}}$.

Moreover, given the Gaussian noise (8), (18) (with the diffusion coefficient proportional to $k T$ ), we have that the mean value of the energy fluctuation is $\delta E_{(T)}=\frac{k T}{2}$. Thence, a non-relativistic scalar particle of mass $m$ owns an energy variance $\Delta E\left(m c^{2}>k T\right)$ : 


$$
\begin{aligned}
\Delta E \approx & \left(<\left(m c^{2}+\delta E_{(T)}\right)^{2}-\left(m c^{2}\right)^{2}>\right)^{1 / 2} \\
\cong & \left(<\left(m c^{2}\right)^{2}+2 \delta E-\left(m c^{2}\right)^{2}>\right)^{1 / 2} \\
& \cong\left(2 m c^{2}<\delta E>\right)^{1 / 2} \cong\left(m c^{2} k T\right)^{1 / 2}
\end{aligned}
$$

from which it follows that:

$$
\left.\Delta E \Delta t>\Delta E \Delta \tau_{\min } \propto \frac{\left(m c^{2} k T\right)^{1 / 2} \lambda_{c}}{c}\right)=\sqrt{2} \hbar,
$$

It is worth noting that the product $\Delta E \Delta \tau$ is constant since the growing of the energy variance with the square root of $T$ is exactly compensated the decrease of the minimum acquisition time $\tau$.

The same result is achieved if we derive the uncertainty relations between the position and momentum of a particle of mass $m$.

If we acquire information about the spatial position of a particle with a precision $\Delta L>\mathcal{L}_{q}$ and the variance of its relativistic momentum $\left(p^{\mu} p_{\mu}\right)^{1 / 2}=m c$ due to the fluctuations reads:

$$
\begin{aligned}
\Delta p \approx & \left(<\left(m c+\frac{\delta E_{(T)}}{c}\right)^{2}-(m c)^{2}>\right)^{1 / 2} \\
& \cong\left(<(m c)^{2}+2 m \delta E-(m c)^{2}>\right)^{1 / 2} \\
& \cong(2 m<\delta E>)^{1 / 2} \cong(m k T)^{1 / 2}
\end{aligned}
$$

it follows that the uncertainty reads:

$$
\left.\Delta L \Delta p>\mathcal{L}_{q}(m k T)^{1 / 2} \propto \lambda_{c}(m k T)^{1 / 2}\right)=\sqrt{2} \hbar
$$

Equating (86) to the uncertainty value such as:

$$
\Delta L \Delta p>\mathcal{L}_{q}(2 m k T)^{1 / 2}=\frac{\hbar}{2}
$$

or

$$
\Delta E \Delta t>\Delta E \Delta \tau_{\min }=\frac{\left(2 m c^{2} k T\right)^{1 / 2} \mathcal{L}_{q}}{c}=\frac{\hbar}{2},
$$

we obtain $\mathcal{L}_{q}=\frac{\lambda_{c}}{2 \sqrt{2}} \approx \frac{\lambda_{c}}{3}$, that represents the physical length below which the quantum coherence is strong.

As far as it concerns the theoretical minimum uncertainty of quantum mechanics, obtainable from the minimum uncertainty (84), (86) in the limit of zero noise, we observe that the quantum deterministic behavior (for $\lambda_{c} \rightarrow \infty$ ) in the low velocity limit (i.e., $c \rightarrow \infty$ ) leads to the equalities:

$$
\Delta \tau_{\min }=\frac{\lambda_{c}}{2 \sqrt{2} c} \text { undefined }
$$

$$
\begin{aligned}
& \Delta E \cong\left(m c^{2} k T\right)^{1 / 2}=\sqrt{2} \frac{\hbar c}{\lambda_{c}} \text { undefined } \\
& \mathcal{L}_{q}=\frac{\lambda_{c}}{2 \sqrt{2}} \rightarrow \infty \\
& \Delta p \cong(m k T)^{1 / 2} \cong \frac{\sqrt{2} \hbar}{\lambda_{c}} \rightarrow 0
\end{aligned}
$$

but the products

$$
\begin{aligned}
& \Delta E \Delta t>\Delta E \Delta \tau_{\text {min }}=\frac{\hbar}{2} \\
& \Delta L \Delta p>\mathcal{L}_{q}(m k T)^{1 / 2}=\frac{\hbar}{2}
\end{aligned}
$$

remain finite and constant.

Thus, if we localize the system in a domain of physical length $\Delta L<\mathcal{L}_{q}$ (where the quantum mechanics is fully realized), due to the increase of the spatial confinement of the wave function, the increase of both the quantum potential energy and its variance follows the minimum uncertainty relation (94).

Since non-locality is confined in domains of physical length of order of $\frac{\lambda_{c}}{2 \sqrt{2}}$ and information about a quantum system cannot be transferred faster than the light speed (otherwise also the uncertainty principle is violated) the local realism is established in the coarse-grained macroscopic physics while the paradox of the "spooky action at a distance" is limited on microscopic distance smaller than $\frac{\lambda_{c}}{2 \sqrt{2}}$ where the quantum mechanics (with both $c \rightarrow \infty$ and $\lambda_{c} \rightarrow \infty$ ) fully apply.

For sake of completeness, it must be observed that the SQHM has been derived for particles with rest mass $m \neq 0$.

As far it concerns the description of the quantum correlations involving photons (for which $\lambda_{c}=\infty$ at whatever temperature $T$ ) it is out of the SQHM and the stochastic generalization of the relativistic quantum hydrodynamic description [29] is needed.

Finally, it is interesting to note that due to the finite light speed, actually, in the deterministic limit of quantum mechanics (i.e., $\mathcal{L}_{q} \rightarrow \infty$ ), the minimum acquisition time of information

$$
\Delta \tau_{\text {min }}=\frac{\mathcal{L}_{q}}{c} \rightarrow \infty
$$

becomes infinite and measures cannot be carried out.

\section{Stochastic Quantum Hydrodynamic Model and the Decoherence Theory}

As part of the SQHM, to perform statistically reproducible measurement processes (which generate the collapse of the wave function) and, therefore, to have the measuring apparatus independent of the measured system (free of 
quantum correlations before and after the measurement), there is the need to have a classical fluctuating global system with finite length of the quantum potential interaction (in which, due to the finite speed of transmission of light and information, it is possible to carry out the measurement in a finite time interval). Therefore, in the SQHM, decoherence and the finite interval of interaction of the quantum potential are preconditions for carrying out the measurement process.

The decoherence theory [46] does not attempt to explain the problem of measurement and the collapse of the wave function. Rather, decoherence provides an explanation for the transition of the system to a statistical mixture of states generated by quantum entanglement leakage with the environment [38], [46]. Furthermore, if for a microscopic system the decoherence process can take a long time, for macroscopic systems, consisting of $\mathrm{n}$ microscopic quantum elements, the decoherence time is $\propto \frac{\tau_{d}}{n}$ that it can be very short. However, the superposition of the global or universal wave function still exists (and remains globally coherent) [46].

This conundrum can be logically accepted using the solution proposed by Poincaré and recently extended to quantum systems [50] which shows that irreversible phenomena can occur in a global system subject to reversible behavior, since the recurrence appears over a much longer time interval than universe all life. (for example, the recurrence time provided by Boltzmann, required for a single $\mathrm{cm}^{3}$ of gas to return to its initial state, has the order of many trillions of digits). Although Madelung's theory and Wigner distributions are intimately related [29] and the two descriptions cannot contradict each other, the classical or quantum nature of the global system is fundamentally a question of interpretation. In other words, it is necessary to discriminate whether the ambient noise is really random or pseudo-random. In computer simulations it is well known that any routine that generates a noise gives pseudo-random output, but no one doubts that this makes a difference.

Decoherence theory explains macroscopicity as a dissipative quantum behavior but the universal system still remains globally quantum. Such quantum "local" and transient decoherence is also described by the pseudopseudo-diffusion of the Madelung's quantum hydrodynamics, but the recurrent initial state is re-obtained by anti-diffusion and anti-decoherent process. Works on the same topic (see for instance [51]) confirm such phenomena. This fact should lead to anti-entropic phenomena "locally" in some places of the global system and at a certain time in the considered "subsystem", but there is no evidence of these anti-entropic phenomena somewhere in the universe and the belief that entropy increase law will cease in the future is somewhat unlikely.

On the contrary, the SQHM approach provides the criterion for the transition from the reversible quantum dynamics to the really irreversible classical ones on macroscopic scale.

The possibility shown by the SQHM to have a classical global system in a space-time with curvature fluctuations of the background metric [42] is a formulation, where the classical mechanics can be seen as the realization of the quantum mechanics on large scale, compatible with the quantum-gravitational description of the universe in which gravity is seen as the source of global decoherence [52]-[54].

\section{Stochastic Quantum Hydrodynamic Model and the Copenhagen Interpretation of Quantum Mechanics}

The path-integral solution of the SQHM is not general but holds in the small noise limit, before a large fluctuation occurs. The path-integral solution of the SQHM model describes the "microscopic stage" of the decoherence process at De Broglie physical length scale.

The SQHM parametrizes the quantum to classical transition by using two physical lengths, $\lambda_{c}$ and $\lambda_{q u}$, seeing the quantum mechanics as the asymptotical behavior for $\lambda_{c} \rightarrow \infty$. Being so, it can furnish additional insight about the measure process.

Even if the measure process can be asymptotically treated as a quantum interaction with the measure apparatus (i.e., $\lim \lambda_{c} \rightarrow \infty$ and $\left.\lim \lambda_{q u} \rightarrow \infty\right)$, marginal effects exist for its realization due to the classical equipment to be used for the experimental management, collection and treatment of the information.

Generally speaking, the decoherence produced during the interaction with the measuring apparatus may be different by that one generated just by the stochastic noise. In order to describe such "marginal" decoherence during the external measuring interaction $V_{\text {ext }}$, the SQHM has to be modified and reads:

$$
\dot{p}_{j}=-m \kappa \dot{q}_{j_{(t)}}-\frac{1}{m} \frac{\partial\left(V+V_{e x t}+V_{q u_{(n)}}\right)}{\partial q_{j}}+m \varpi_{j(q, t, T)}
$$

where

$V_{\text {ext }}=0$ for $t<t_{0} \cup t>t_{0}+\Delta \tau$

In principle, the marginal decoherence with characteristic time $\tau_{r}$, may take part to the measurement if $\tau_{r}$ is comparable with $\Delta \tau$. The absence of marginal effects is included as the particular case of sufficiently fast measurement with $\Delta \tau \ll \tau_{r}$.

From the general point of view, the SQHM shows that, the steady state after the relaxation depends on its initial configuration

$\left|\psi_{(q, t)}>=\sum_{n} a_{n} e^{\frac{E_{n}\left(t-t_{0}\right)}{\hbar}}\right| \psi_{n(q)}>$

at the moment $t_{0}$, allowing the system to possibly reach whatever eigenstate of the superposition.

Since the quantum superposition of energy eigenstates possesses a cyclic evolution with period

$\mathrm{T}=\frac{2 \pi \hbar}{E_{\min }}$ 
where $E_{\text {min }}$ is its lowest energy eigenvalue, the probability of relaxation to the $i$-th energy eigenstate for the SQHM model reads:

$$
P\left(\psi \rightarrow \psi_{i}\right)=\lim _{N \rightarrow \infty} \frac{N_{i}}{N}
$$

where $N$ is the number of time intervals $\Delta t_{j}=\frac{\mathrm{T}}{N} ; j=1, \ldots, N \quad$ centered around $t_{0_{i}} \quad$ (with $\left.0 \leq t_{0}<\mathrm{T}\right)$ in which the system starts to evolve in presence of marginal fluctuations, and $N_{i}$ is the number of times in which the final stationary state is the $i$-th energy eigenstate.

For the Copenhagen interpretation of quantum mechanics, the measurement is a process that produces the wave function collapse and the outcome (e.g., the energy value $E_{n}$ for the state (97)) is described by the transition probability that reads:

$$
\tilde{P}\left(\psi \rightarrow \psi_{n}\right)=\frac{\left|a_{n}\right|^{2}}{\sum_{k=1}^{t}\left|a_{k}\right|^{2}},
$$

Given marginal quantum-stochastic effects, generating relaxation with characteristic time $\tau_{r}$ and measure with a duration $\Delta \tau$, for sufficiently fast measurement $\Delta \tau \ll \tau_{r}$, the final output reads:

$$
\begin{aligned}
& \lim _{\frac{\tau_{r}}{\Delta \tau} \rightarrow \infty} \tilde{P}_{(t o t)}\left(\psi \rightarrow \psi_{n}\right)=\tilde{P}\left(\psi \rightarrow \psi_{i}\right) P\left(\psi_{i} \rightarrow \psi_{n}\right) \\
= & \sum_{i} \frac{\left|a_{i}\right|^{2}}{\sum_{k=1}^{t}\left|a_{k}\right|^{2}} \lim _{N \rightarrow \infty} \frac{N_{n}}{N} \\
= & \sum_{i} \frac{\left|a_{i}\right|^{2}}{\sum_{k=1}^{t}\left|a_{k}\right|^{2}} \delta_{i n}=\frac{\left|a_{n}\right|^{2}}{\sum_{k=1}^{t}\left|a_{k}\right|^{2}}
\end{aligned}
$$

Showing that the marginal quantum irreversibility does not affect the measure (i.e., the Born's rule). Conversely, for sufficiently fast induced marginal decoherence with $\Delta \tau \gg \tau_{r}$ , it follows that:

$$
\begin{aligned}
& \lim _{\frac{\tau_{r}}{\Delta \tau} \rightarrow 0} \tilde{P}_{(t o t)}\left(\psi \rightarrow \psi_{n}\right)=\sum_{i} P\left(\psi \rightarrow \psi_{i}\right) \tilde{P}\left(\psi_{i} \rightarrow \psi_{n}\right) \\
& =\sum_{i} \lim _{N \rightarrow \infty} \frac{N_{i}}{N} \frac{\left|a_{n\left(\psi_{i}\right)}\right|^{2}}{\sum_{k=1}^{t}\left|a_{k\left(\psi_{i}\right)}\right|^{2}}=\sum_{i} \lim _{N \rightarrow \infty} \frac{N_{i}}{N} \delta_{i n} \\
& =\lim _{N \rightarrow \infty} \frac{N_{n}}{N}
\end{aligned}
$$

Thus, if the measure on a quantum states (obeying the Born's rule) ends with a decoherent statistical mixture so that $\Delta \tau>\tau_{r}$, it follows that:

$$
\frac{\left|a_{n}\right|^{2}}{\sum_{k=1}^{t}\left|a_{k}\right|^{2}}=\lim _{N \rightarrow \infty} \frac{N_{n}}{N}
$$

Numerical simulations are in progress to validate (103).

Given the Copenhagen interpretation of the quantum mechanics in which the wave function collapse produces the transition from a coherent to an inconsistent state (in which the terms out-diagonal of the density matrix are null) and the decoherence approach that spontaneously causes the transition from a coherent state to a decoherent one (in which the off-diagonal terms of the density matrix are asymptotically null after the relaxation process), the inspection of identity (103) can furnish insight about the wave function collapse. That is, how quickly the measured quantum system loses correlation with its initial state during the measure and if the measuring time is correlated to such property. The validation of (103) would imply that the measurement cannot be performed faster than the measured system does to reach the relaxed state.

\section{E. Fields of Application of the SQHM}

The theory that describes how the quantum entanglement extends itself up to a certain distance and how to improve the quantum coherence, can lead to important advancements in the development of materials for superconductors at high temperature and systems to realizing Qbits for quantum computing. The description of kinetics of complex chemical reactions at the edge of quantum to classical regime and the study of phenomena occurring at the mesoscale can also take advantage from the SQHM.

\section{CONCLUSION}

By using the Madelung quantum hydrodynamic representation in fluctuating vacuum, the stochastic evolution of quantum microscopic systems is derived. The general pathintegral solution, also for non-linear systems, is given in a recursive form. The model contains the reversible quantum mechanics as the deterministic limit of the theory.

When the quantum eigenstates are submitted to fluctuations, their stationary configurations are slightly perturbed but remain stationary and close to those ones of quantum mechanics. On the contrary, the evolution of the superpositions of states are deeply perturbed and relax to stationary configurations of one of the quantum eigenstates.

The SQHM, where the amplitude of the stochastic noise depends by the vacuum De Broglie physical length, naturally introduces the concept of the range of action of the non-local quantum potential which depends by the strength of the Hamiltonian interaction, showing that systems, sufficiently weakly interacting, can lead to the classical mechanics on coarse-grained macroscopic limit.

Since the SQHM gives a unified approach for quantum and classical behaviors, it establishes a connection between the 
uncertainty principle of quantum mechanics and the locality of macroscopic classical description. The minimum uncertainty, in the large-scale classical reality, is warranted if interactions and information do not travel faster than the speed of light, showing that the macroscopic locality and the relativistic postulate are compatible with the microscopic non-local quantum interactions:

The generalized minimum uncertainty of the SQHM leads to the minimum uncertainty principle of quantum mechanics in the limit of zero noise.

The SQHM and the decoherence theory do not contradict themselves and differ just by an interpretational point of view: The SQHM gives a criterion in order to discriminate when the macroscopic dissipative quantum global system can really acquire the classical behavior, while the decoherence theory assumes that even if the global system is quantum, it locally mimics the classical behavior in a way that it is not possible to discriminate between the two situations since the recurrence time is of order of magnitude very much bigger than the life time of the universe.

The SQHM, furnishes the alternative interpretation of decoherence, addressing it as consequence of gravity fluctuations in a quantum space-time, which can lead to a really classical global system.

The SQHM shows that the decoherence is necessary in order for realizing a measure and that it possibly also contributes during its execution.

\section{APPENDIX}

\section{A. Appendix A}

Quantum mechanics as (immaginary-time) stochastic process.

The Schrödinger equation:

$i \hbar \frac{\partial \psi}{\partial t}=-\frac{\hbar^{2}}{2 m} \frac{\partial^{2} \psi}{\partial q_{i} \partial q_{i}}+U_{(q)} \psi$

in the quantum integral path representation, is equivalent to the FPE [46] that in the unidimensional case reads:

$$
\frac{\partial P_{\left(q, t \mid q_{a}, t_{a}\right)}}{\partial t}=-\frac{\partial K(q) P_{\left(q, t \mid q_{a}, t_{a}\right)}}{\partial q}+D \frac{\partial^{2} P_{\left(q, t \mid q_{a}, t_{a}\right)}}{\partial q^{2}}
$$

where the solution

$P_{\left(q, t \mid q_{a}, t_{a}\right)}=\sqrt{\frac{P_{s(q)}}{P_{s\left(q_{a}\right)}}} \int_{q_{a}}^{v} D q e^{\left[-\frac{\int_{t_{a}}^{t} d t\left(\frac{1}{2} \dot{q}_{(t)}^{2}+\frac{1}{2} K_{(q)}+D K^{\prime}(q)\right)}{2 D}\right]}$

where

$$
\begin{aligned}
P_{S(q)} & =P\left(q, q_{-\infty} \mid t-t_{-\infty}, t_{-\infty}\right) \\
& =\lim _{t_{0} \rightarrow-\infty} N \exp \frac{1}{D} \int_{q_{-\infty}}^{q}<\dot{q}>_{\left(q_{\left(t, t_{0}\right)}^{\prime}\right)} d q^{\prime} \\
& =N \exp \frac{1}{D} \int_{q_{-\infty}}^{q} K\left(q^{\prime}\right) d q^{\prime}
\end{aligned}
$$

and

$$
K_{(q)}^{\prime}=\frac{\partial K_{(q)}}{\partial q}
$$

leads to the imaginary time $\tau=i t$ evolution amplitude

$$
\begin{aligned}
\psi_{(q, t)} & =\psi_{(q, 0)} \int D q e^{\left[-\frac{1}{\hbar} \int_{0}^{-i \tau} d \tau\left(\frac{M}{2} \dot{q}_{(\tau)}^{2}+V_{(q(\tau))}\right)\right]} \\
& =\sqrt{\frac{P_{s\left(q_{a}\right)}}{P_{s(q)}}} P_{\left(q, t \mid q_{a}, t_{a}\right)}
\end{aligned}
$$

By comparing (A.6) with (A.2) we can see that a quantum particle subject to the potential:

$$
V_{(q)}=\frac{m}{2} K_{(q)}^{2}+\frac{\hbar}{2} \frac{\partial K_{(q)}}{\partial q}
$$

is equivalent to a Brownian particle with mass $m$ and friction coefficient $\beta=\kappa m$ obeying to the SDE:

$\dot{q}_{j}=-\frac{1}{\beta} \frac{\partial U_{(q)}}{\partial q_{j}}+D_{j k}^{1 / 2} \xi_{k}$

where the potential $U_{(q)}$ reads $\frac{\partial U_{(q)}}{\partial x}=-m \kappa K_{(q)}$ and where $D_{i k}=\frac{\hbar}{2 m} \delta_{j k}$.

The "diffusional" effect of the imaginary-time stochastic process (A.8) can be derives by studying the motion of the conserved quantum mass density $n=|\psi|^{2}$ [29], [45]. This can be easily done in the quantum hydrodynamic formalism that describes the wave function

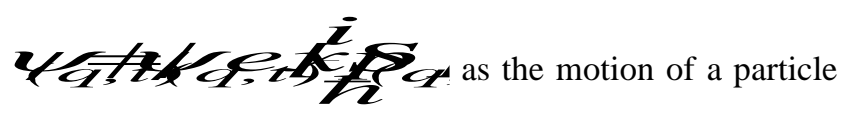
density $n_{(q, t)}=|\psi|_{(q, t)}^{2} \quad$ with $\quad$ velocity $\left.\quad \dot{q}_{i}=\frac{1}{m} \frac{\partial S_{(q, t)}}{\partial q_{i}}\right)$ governed by the equations [29], [45]"

$$
\begin{aligned}
& \partial_{t} n_{(q, t)}+\frac{\partial\left(n_{(q, t)} \dot{q}\right)}{\partial q_{i}}=0, \\
& \dot{q}_{i}=\frac{1}{m} \frac{\partial S_{(q, t)}}{\partial q_{i}}=\frac{p_{i}}{m}, \\
& p_{i}=-\frac{\partial\left(H+V_{q u}\right)}{\partial q_{i}},
\end{aligned}
$$


where, for a non-relativistic particle in an external potential,

$H=\frac{p_{i} p_{i}}{2 m}+V_{(q)}$

and where

$S=\int_{t_{0}}^{t} d t\left(\frac{p_{i} p_{i}}{2 m}-V_{(q)}-V_{q u}\right)$

where $V_{q u}$ is the quantum pseudo-potential that reads:

$V_{q u}=-\left(\frac{\hbar^{2}}{2 m}\right) n^{-1 / 2} \frac{\partial}{\partial q_{j}} \frac{\partial}{\partial q_{j}} n^{1 / 2}$,

Expression (A.14) leads to the motion equation:

$\dot{q}=\frac{1}{m} \frac{\partial S}{\partial q_{i}}=\frac{1}{m} \int_{t_{0}}^{t} d t\left(\frac{\partial}{\partial q_{i}} \frac{p_{i} p_{i}}{2 m}-\nabla\left(V_{(q)}-V_{q u}\right)\right)$

$=-\frac{1}{m} \int_{t_{0}}^{t} d t\left(\frac{\partial\left(V_{(q)}-V_{q u}\right)}{\partial q_{i}}\right)$

and to the stationary equilibrium condition (i.e., $\dot{q}=0$ ):

$-\frac{1}{m} \int_{t_{0}}^{t} d t\left(\frac{\partial\left(V_{(q)}-V_{q u}\right)}{\partial q_{i}}\right)=0$

that, by using (A.14), reads:

$\frac{\partial}{\partial q_{i}}\left(V_{(q)}+\frac{\hbar^{2}}{2 m} n^{-1 / 2} \frac{\partial}{\partial q_{j}} \frac{\partial}{\partial q_{j}} n^{1 / 2}\right)=0$

Moreover, by comparing (A.17), for instance, with the Fick equation, we can see that the quantum imaginary stochastic process (A.8) generates a "pseudo-diffusional" process driven by the quantum potential:

$$
V_{q u} \propto n^{-1 / 2} \frac{\partial}{\partial q_{j}} \frac{\partial}{\partial q_{j}} n^{1 / 2}
$$

(in a similar way of the chemical potential $\propto \ln n$ for the classical diffusion).

The (real time) thermal stochasticity and the quantum (immaginary time) stochastic process show that the stationary states (i.e., $\dot{q}=0$ ) happens when the force of the fluctuations-generated potential exactly counterbalances, point by point, that one given by the Hamiltonian potential $V_{(q)}$.

The basic difference between the two is that, in the quantum case, we may have more than one stationary state (i.e., the quantum eigenstates) exist whose quantum potential counter-balances the hamiltonian potential (see Appendix B ref. [36]).

Therefore, even if an analogy exists between the real-time stochastic process and the imaginary-time one, they own important differences.

For instance, the quantum "imaginary-time" Brownian process for the Gaussian mass-distribution packet leads to the time law for the variance:

$\Delta q^{2} \propto t^{2}$,

while the spreading of the same mass distribution, submitted to thermal fluctuations, follows the variance time-law:

$\Delta q^{2} \propto t$

Furthermore, it must be observed that the "real-time" stochastic dynamics are dissipative while the "imaginarytime" stochastic dynamics of quantum mechanics are reversible and "deterministic".

This dissipative-to-conservative change of characteristics in passing from a real to an imaginary parameter is similar (with inverted parts) to what happens in the rheology of elastic solids where real elastic constants lead to conservative elastic dynamics while imaginary elastic constants describe the viscous dissipative relaxation in solids [54].

In the quantum deterministic evolution there is no loss of information and there is not relaxation: Therefore, the superposition of states (with their complex configuration) are maintained a long time and never relax to a stationary equilibrium configuration.

The present work is aimed to describe what happens in the general case when the two stochastic processes are contemporarily present.

\section{B. Appendix $B$}

In the case of linear systems:

$V_{(q)}=\frac{m \omega^{2}}{2} q^{2}$

the equilibrium condition leads to:

$\frac{\hbar^{2}}{4 m} \frac{\partial\left(\left(\frac{\partial^{2} \ln n}{\partial q^{2}}\right)+\frac{1}{2}\left(\frac{\partial \ln n}{\partial q}\right)^{2}\right)}{\partial q}=m \kappa D \frac{\partial \ln n}{\partial q}+m \omega^{2} q$

that is satisfied by the solution $n=n_{0} \exp \left[-\frac{q^{2}}{\Delta q^{2}}\right]$, where $n_{0(q)}$ is defined by the relation. 


$$
\begin{aligned}
& \frac{\hbar^{2}}{4 m^{2}} \frac{\partial\left(\left(\frac{\partial^{2}}{\partial q^{2}}\left(\ln n_{0}-\frac{q^{2}}{\Delta q^{2}}\right)\right)+\frac{1}{2}\left(\frac{\partial}{\partial q}\left(\ln n_{0}-\frac{q^{2}}{\Delta q^{2}}\right)\right)^{2}\right)}{\partial q} . \\
& =\kappa D \frac{\partial\left(\ln n_{0}-\frac{q^{2}}{\Delta q^{2}}\right)}{\partial q}+\omega^{2} q
\end{aligned}
$$

For the fundamental eigenstate:

$$
\lim _{T \rightarrow 0} n_{0}{ }^{1 / 2} \exp \left[-\frac{q^{2}}{2 \Delta q^{2}}\right]=\left(\frac{1}{4 \pi} \frac{m \omega}{\hbar}\right)^{1 / 4} \exp \left[-\frac{q^{2}}{2 \Delta q_{0}^{2}}\right]
$$

from (B.3) it follows that:

$$
\frac{1}{\Delta q^{2}}=-\frac{m^{2}}{\hbar^{2}} \kappa D+\frac{m \omega}{\hbar} \sqrt{1+\left(\frac{m}{\hbar \omega} \kappa D\right)^{2}}
$$

that near the quantum mechanics (i.e., $D \rightarrow 0, \kappa \rightarrow 0$ with $\omega \gg \frac{m}{\hbar} \kappa D$ ) leads to:

$\frac{1}{\Delta q^{2}} \cong \frac{m \omega}{\hbar}-\frac{m^{2}}{\hbar^{2}} \kappa D+\frac{1}{2} \frac{m^{3}}{\hbar^{3} \omega}(\kappa D)^{2}$

$\cong \frac{m \omega}{\hbar}-\frac{m^{2}}{\hbar^{2}} \kappa D \cong \frac{m \omega}{\hbar}\left(1-2 \alpha \frac{k T}{\hbar \omega}\right)$

and to the distribution:

$n_{e q}=n_{0} \exp \left[-\frac{m \omega}{\hbar}\left(1-2 \alpha \frac{k T}{\hbar \omega}\right) q^{2}\right]$

where

$n_{0}=\left[\frac{1}{4 \pi} \frac{m \omega}{\hbar}\left(1-2 \alpha \frac{k T}{\hbar \omega}\right)\right]^{1 / 2}$.

From (38) it can be observed that, within the limit of small thermal fluctuations, the density distribution of the fundamental eigenstate (37) does not lose its Gaussian form and acquires a small enlargement of its variance:

$\Delta q^{2}=\Delta q_{0}^{2}\left(1+2 \alpha \frac{k T}{\hbar \omega}\right)$.

where

$\Delta q_{0}^{2}=\left(\frac{\hbar}{m \omega}\right)$. is the variance of the deterministic quantum state. As initially stated, the result (B.7) satisfies condition (27).

Moreover, in presence of fluctuation, the energy $E_{0}$ of the fundamental stationary state reads:

$$
\begin{aligned}
E_{0} & =<\psi_{0}|H| \psi_{0}>=\int_{-\infty}^{\infty} n_{(q, t)}\left[\frac{m}{2} \dot{q}^{2}+\frac{m \omega^{2}}{2} q^{2}+V_{q u}\right] d q \\
& =\int_{-\infty}^{\infty} n_{(q, t)}\left[\frac{m \omega^{2}}{2} q^{2}+V_{q u}\right] d q \\
& =\int_{-\infty}^{\infty} n_{(q, t)}\left[\frac{m \omega^{2}}{2} q^{2}-\frac{m \omega^{2}}{2}\left(1-2 \alpha \frac{k T}{\hbar \omega}\right) q^{2}+\left(n+\frac{1}{2}\right) \hbar \omega\right] d q \\
& =\int_{-\infty}^{\infty} n_{(q, t)}\left[\alpha \frac{\omega m k T}{\hbar} q^{2}+\frac{\hbar \omega}{2}\right] d q \\
& =\frac{\hbar \omega}{2}+\frac{\alpha k T}{\pi^{1 / 2}}\left(\frac{m \omega}{\hbar}\right)^{\frac{3}{2}} \int_{-\infty}^{\infty} q^{2} e^{\left[-\left(\frac{m \omega}{\hbar}-\frac{2 \alpha m k T}{\hbar^{2}}\right) q^{2}\right]} d q \\
& =\frac{\hbar \omega}{2}+\alpha \frac{\omega m k T}{\hbar}\left(\frac{1}{1+2 \alpha \frac{k T}{\hbar \omega}}\right) \Delta q^{2} \\
& \cong \frac{\hbar \omega}{2}+\alpha k T
\end{aligned}
$$

showing an energy increases of $\alpha k T$.

As far as it concerns the energy variance of the fundamental stationary state:

$\Delta E_{0}=<\psi_{0}\left|\left(E-E_{0}\right)^{2}\right| \psi_{0}>^{1 / 2}$,

in presence of fluctuation, it reads:

$$
\begin{aligned}
\left(\Delta E_{0}\right)^{2} & =\int_{-\infty}^{\infty} n_{(q, t)}\left[\left(\frac{m}{2} \dot{q}^{2}+\frac{m \omega^{2}}{2} q^{2}+V_{q u}\right)-\left(\frac{\hbar \omega}{2}+\alpha k T\right)\right]^{2} d q \\
& =\int_{-\infty}^{\infty} n_{(q, t)}\left[\alpha \frac{\omega m k T}{\hbar} q^{2}-\alpha k T\right]^{2} d q \\
& =(\alpha k T)^{2} \int_{-\infty}^{\infty} n_{(q, t)}\left[\frac{\omega m}{h} q^{2}-1\right]^{2} d q \\
& =\alpha k T\left(-1+\frac{\omega m}{\hbar} \int_{-\infty}^{\infty} n_{(q, t)}\left(2 q^{2}+\frac{\omega m}{\hbar} q^{4}\right) d q\right) \\
& \cong(\alpha k T)^{2}\left(-1+1+\left(\frac{\omega m}{\hbar}\right)^{2} \int_{-\infty}^{\infty} n_{(q, t)} q^{4} d q\right) \\
& \cong \frac{3}{8}(\alpha k T)^{2}
\end{aligned}
$$


that allows to derive the dissipation parameter $\alpha$ from the energy variance of the fundamental state such as:

$\alpha \cong \sqrt{\frac{8}{3}} \frac{\Delta E_{0}}{k T}$

For higher eigenstates (see ref. [36]) the eigenvalues of the Hamiltonian read:

$$
E_{j} \cong\left(j+\frac{1}{2}\right) \hbar \omega-\left(1+\Delta_{j}\right) \alpha k T
$$

where

$$
\Delta_{j}=\frac{\Delta q^{2}}{\left(\Delta q_{j}^{2}-\Delta q_{j-1}^{2}\right)}-1
$$

where $\Delta q_{j}^{2}$ is the wave function variance of the $j$-th eigenstate and $\Delta q^{2}$ is the variance of the fundamental one.

It is noteworthy to see that the parameter $\alpha$ can be also experimentally evaluated by the measure of the energy gap $\Delta E_{j}=E_{j}-E_{j-1}$ between eigenstates through the relation:

$$
\alpha \cong \frac{\Delta q^{2}}{\left(\Delta q_{j}^{2}-\Delta q_{j-1}^{2}\right)} \frac{\hbar \omega-\left(E_{j+1}-E_{j}\right)}{k T}
$$

\section{Appendix $C$}

The condition $\lim _{\Delta t \rightarrow 0} \lim _{D \rightarrow 0} \varepsilon \rightarrow 0$ is warranted by the existence of the deterministic continuous limit that by (49) implies that:

$$
\begin{aligned}
& \lim _{D \rightarrow 0} \mathscr{P}\left(q_{k}, q_{k-1} \mid \Delta t,(k-1) \Delta t\right) \\
& =\lim _{D \rightarrow 0 \Delta t \rightarrow 0} 4 \sqrt{\pi D \Delta t} e^{\left[-\left(\dot{q}_{k-1}-<\dot{\bar{q}}_{k-1}>\right)^{2} \Delta t+2 D \frac{\partial<\dot{\bar{q}}_{k-1}>}{\partial \bar{q}_{k-1}} \Delta t\right]} \\
& 4 D \\
& =\lim _{\Delta t \rightarrow 0 D \rightarrow 0} 4 \sqrt{\pi D \Delta t} e^{\left[-\left(\dot{q}_{k-1}-<\dot{\bar{q}}_{k-1}>\right)^{2} \Delta t+2 D \frac{\partial<\dot{q}_{k-1}>}{\partial \bar{q}_{k-1}} \Delta t\right]} \\
& =\lim _{\Delta t \rightarrow 0} \Delta t^{-1 / 2} \delta\left(\left(\dot{q}_{k-1}-<\dot{\bar{q}}_{k-1}>\right)^{2} \Delta t\right) \\
& =\delta\left(\lim _{\Delta t \rightarrow 0}\left(\dot{q}_{k-1}-<\dot{\bar{q}}_{k-1}>\right)^{2}\right)
\end{aligned}
$$

and that

$$
\lim _{\Delta t \rightarrow 0} \lim _{D \rightarrow 0} \dot{q}_{k-1}=\lim _{\Delta t \rightarrow 0}<\dot{\bar{q}}_{k-1}>=<\dot{q}_{k-1}>.
$$

\section{Appendix D}

In quantum measurements the preparation of the experiment needs initial conditions where the system and the apparatus are well defined. This can be done by assuming they are decoupled (i.e., being at infinity) with the tacit assumption of no interaction at all. Nonetheless, since a quantum system acts as a whole (i.e., the non-local quantum potential interaction is present everywhere and thus even at infinite distance) the null-interaction can be strictly assumed true only if the quantum non-local potential has a finite range of action as in presence of random fluctuations (noise shield). Thus, the marginal decoherence cannot be disregarded in the measurement process.

\section{REFERENCES}

[1] Young, T., Phil. Trans. R. Soc. Lond. 94, 1 (1804).

[2] Einstein, A., Podolsky, B. and Rosen, N. (1935) Can QuantumMechanical Description of Physical Reality Be Considered Complete? Physical Review, 47, 777-780. http://dx.doi.org/10.1103/PhysRev.47.777

[3] Bell, J.S., On the Einstein- Podolsky-Rosen paradox, Physics, 1:195200 (1964).

[4] Feynmann, R. P., The Feynman Lectures on Physics, Volume 3 , (AddisonWesley, 1963).

[5] Auletta, G., Foundations and Interpretation of Quantum Mechanics, (World Scientific, 2001).

[6] Greenstein, G. .and Zajonc, A. G., The Quantum Challenge, (Jones and Bartlett Publishers, Boston, 2005), 2nd ed.

[7] Shadbolt, P., J. Mathews, C. F., Laing, A., and OBrien, J. L., Nature Physics 10, 278 (2014).

[8] Josson, C., Am. J. Phys, 42, 4 (1974).

[9] Zeilinger, A., Gahler, R., Shull, C.G., Treimer W., and Mampe, W., Rev. Mod. Phys. 60, 1067 (1988).

[10] Carnal, O., and Mlynek, J., Phys. Rev. Lett. 66, 2689 (1991).

[11] Schollkopf, W.and Toennies, J.P., Science 266, 1345 (1994).

[12] Arndt, M., Nairz, O., Vos-Andreae, J., Keller, C., van der Zouw, G. and Zeilinger, A., Nature 401, 680 (1999).

[13] O. Nairz, M. Arndt and A. Zeilinger, Am. J. Phys. 71, 319 (2003).

[14] Born, M., The statistical interpretation of quantum mechanics -

[15] Nobel Lecture, December 11, 1954.

[16] Ruggiero P. and Zannetti, M., Phys. Rev. Lett. 48, 963 (1982).

[17] Ghirardi, G.C., Collapse Theories, Edward N. Zalta (ed.), The Stanford Encyclopedia of Philosophy (Fall 2018 Edition).

[18] Pitaevskii, P., Vortex lines in an Imperfect Bose Gas, Soviet Physics JETP. 1961;13(2):451-454.

[19] Everette, H., Rev. Mod. Phys. 29, 454 (1957).

[20] Vaidman, L., Many-Worlds Interpretation of Quantum Mechanics, Edward N. Zalta (ed.), The Stanford Encyclopedia of Philosophy (Fall 2018 Edition).

[21] Bohm, D., Phys. Rev. 85, 166 (1952).

[22] Goldstein, S., Bohmian Mechanics, Edward N. Zalta (ed.), The Stanford Encyclopedia of Philosophy (Summer 2017 Edition).

[23] Lombardi O., and Dieks, D., Modal Interpretations of Quantum Mechanics, Edward N. Zalta (ed.), The Stanford Encyclopedia of Philosophy (Spring 2017 Edition).

[24] Laudisa, F., and Rovelli, C.. Relational Quantum Mechanics, Edward N. Zalta (ed.), The Stanford Encyclopedia of Philosophy (Summer 2013 Edition).

[25] Griffiths, R.B., Consistent Quantum Theory, Cambridge University Press (2003)

[26] Cramer, J.G., Phys. Rev. D 22, 362 (1980).

[27] Cramer, J.G., The Quantum Handshake: Entanglement, Non-locality and Transaction, Springer Verlag (2016).

[28] Von Baeyer, H. C., QBism: The Future of Quantum Physics, Cambridge, Harvard University Press, (2016)

[29] Madelung, E.: Z. Phys. 40, 322-6 (1926).

[30] Bialyniki-Birula, I., Cieplak, M., Kaminski, J., "Theory of Quanta", Oxford University press, Ny, (1992) 87-111.

[31] Weiner, J.H., Statistical Mechanics of Elasticity (John Wiley \& Sons, New York, 1983), p. 315-7.

[32] Lidar, D. A.; Chuang, I. L.; Whaley, K. B. (1998). "Decoherence-Free Subspaces for Quantum Computation". Physical Review Letters. 81 (12): 2594-2597.

[33] Tsekov, R., Bohmian mechanics versus Madelung quantum hydrodynamics, arXiv:0904.0723v8 [quantum-phys] (2011).

[34] Gardner, C.L. (1994) The Quantum Hydrodynamic Model for Semiconductor Devices. SIAM Journal on Applied Mathematics, 54, 409-427. http://dx.doi.org/10.1137/S0036139992240425. 
[35] Bousquet, D., Hughes, K.H., Micha, D.A. and Burghardt, I. (2001) Extended Hydrodynamic Approach to Quantum- Classical Nonequilibrium Evolution. I. Theory. The Journal of Chemical Physics, 134, 064116. http://dx.doi.org/10.1063/1.3553174.

[36] Chiarelli, P. (2013) Quantum to Classical Transition in the Stochastic Hydrodynamic Analogy: The Explanation of the Lindeman Relation and the Analogies between the Maximum of Density at Lambda Point and That at the Water-Ice Phase Transition. Physical Review \& Research International, 3, 348-366.

[37] Chiarelli, S. and Chiarelli, P. Stability of quantum eigenstates and kinetics of wave function collapse in a fluctuating environment (2020) arXiv:2011.13997v1 [quant-ph].

[38] Chiarelli, P. (2013) Can Fluctuating Quantum States Acquire the Classical Behavior on Large Scale? Journal of Advanced Physics, 2, 139-163.

[39] W. Zurek and J.P. Paz, Decoherence, chaos and the second law, arXiv:gr-qc/9402006v2 3 Feb 1994.

[40] Mariano, A., Facchi, P. and Pascazio, S. (2001) Decoherence and Fluctuations in Quantum Interference Experiments. Fortschritte der Physik, 49, 1033-1039.

[41] Cerruti, N.R., Lakshminarayan, A., Lefebvre, T.H., Tomsovic, S.: Exploring phase space localization of chaotic eigenstates via parametric variation. Phys. Rev. E 63, 016208 (2000). http://dx.doi.org/10.1103/PhysRevE.63.016208.

[42] Wang, C., Bonifacio, P., Bingham, R., Mendonca, J., T., Detection of quantum decoherence due to spacetime fluctuations, $37^{\text {th }}$ COSPAR Scientific Assembly. Held 13-20 July 2008, in Montréal, Canada., p. 3390.

[43] Chiarelli, S. and Chiarelli, P. (2020) Stochastic Quantum Hydrodynamic Model from the Dark Matter of Vacuum Fluctuations: The Langevin-Schrödinger Equation and the Large-Scale Classical Limit. Open Access Library Journal, 7, 1-36. doi: 10.4236/oalib.1106659.

[44] Nelson, E., Derivation of the Schrödinger Equation from Newtonian Mechanics, Phys. Rev. 150, 1079 (1966).

[45] Klinert, H., Pelster, A., Putz, M. V., Variational perturbation theory for Marcov processes, Phys. Rev. E 65, 066128 (2002).

[46] Gardiner, C.W., Handbook of Stochastic Method, 2 nd Edition, Springer, (1985) ISBN 3-540-61634.9, pp. 331-41.

[47] Rumer, Y.B. and Ryvkin, M.S. (1980) Thermodynamics, Statistical Physics, and Kinetics Mir Publishers, Moscow, 269.

[48] W. Zurek: Decoherence and the Transition from Quantum to Classical_Revisited (https://arxiv.org/pdf/quantph/0306072.pdf), Los Alamos Science Number 27 (2002).

[49] Bressanini D. "An accurate and compact wave function for the $4 \mathrm{He}$ dimer", EPL 2011;96.

[50] Chiarelli, P., The Density Maximum of $\mathrm{He}_{4}$ at the Lambda Point Explained by the Quantum Hydrodynamic Analogy. American Journal of Physical Chemistry. Vol. 2, No. 6, 2013, pp. 122-131. doi: 10.11648/j.ajpc.20130206.12.

[51] Chiarelli, P., Minimum Black hole mass, Physical Science International Journal, 9(4): 1-25, 2016.

[52] Kowalski A. M., Plastino A., Decoherence, Anti-decoherence, and Fisher Information, Entropy, to be published. 2021.

[53] Venuti, L., C., The recurrence time in quantum mechanics arXiv: $1509.04352 \mathrm{v} 2$ [quant-ph].

[54] Bassi A., et al. "Gravitational decoherence", Class. Quantum Grav. 34 193002, 2017.

[55] Pfister, C., Kaniewski, J., Tomamichel, M., et al. A universal test for gravitational decoherence. Nat Commun 7, 13022 (2016). https://doi.org/10.1038/ncomms13022.

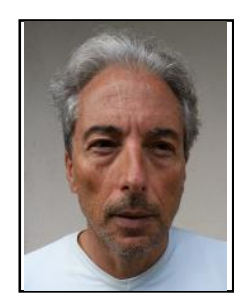

P. Chiarelli Since 1988 has been a CNR Researcher and since 1998 Professor at the Engineering Faculty of the University of Pisa. He was Visiting Associate at the National Institute of Health at Bethesda (USA); Visiting Scientist at the Agency of Industrial Science and Technology of MITI in Tsukuba (Japan), at the Department of Chemistry of the University of Budapest and at the Department of Chemistry of the University of Dresden. 